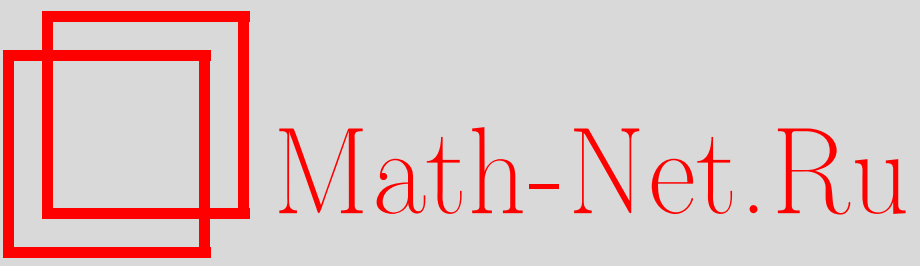

В. И. Сердобольский, Теория существенно многомерного статистического анализа, УМН, 1999, том 54, выпуск 2, 85-112

DOI: https://doi.org/10.4213/rm133

Использование Общероссийского математического портала Math-Net.Ru подразумевает, что вы прочитали и согласны с пользовательским соглашением

http://www.mathnet.ru/rus/agreement

Параметры загрузки:

IP : 3.85 .5 .30

26 апреля 2023 г., 17:12:46 
УДК 519.2

\section{ТЕОРИЯ СУЩЕСТВЕННО МНОГОМЕРНОГО СТАТИСТИЧЕСКОГО АНАЛИЗА}

\section{В.И. СЕРДОБОЛЬСКИЙ \\ СОДЕРЖАНИЕ}

Введение

1. Метод растущей размерности в задачах многомерного статистичес-

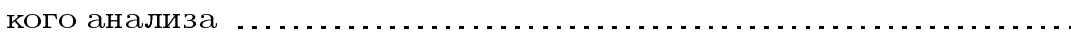

2. Предельная спектральная теория выборочных ковариационных мат-

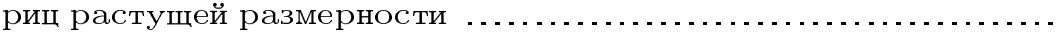

3. Главные части резольвенты и спектральных функций выборочных ко-

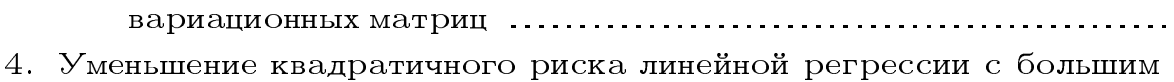
числом случайных предикторов ............................. 99

5. Улучшение линейного дискриминантного анализа . . . . . . . . . . . . . . 104

6. Нормализация при оценке качества многомерных статистических процедур ............................................. 107

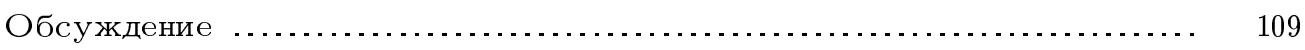

Список литературы . . . . . . . . . . . . . . . . . . . . . . . . . . . . . . . . 111

\section{Введение}

Классические математические исследования по многомерному статистическому анализу сводились к вычислению некоторых точных распределений и их функций в предположении о нормальности наблюдений. Традиционные асимптотические методы статистики (см., например, [1]) ориентированы на одномерные или маломерные задачи. Их формальное перенесение на многомерные задачи (путем замены скаляров на векторы и матрищы) без учета эффектов накопления погрешностей оценок большого числа параметров не привнесло в многомерный статистический анализ ни новых методов, ни новых результатов. Можно сказать, что центральные проблемы многомерного статистического анализа остаются нерешенными. Неулучшаемые статистические процедуры, за некоторым исключением, построить не удается (см. [2; гл. 8]). Простейшая задача оценивания векторов математических ожиданий с наименьшим квадратичным риском решена только для нормальных векторов

Работа выполнена при поддержке Российского фонда фундаментальных исследований (гранты № № 96-01-01574 и 98-01-00781). 
с независимыми компонентами. Стандартные линейные методы многомерного статистического анализа в зависимости от данных могут давать плохо обусловленные решения или не давать никакого решения (если выборочная ковариационная матрица вырождена). В случае, когда объем выборки ненамного превышает размерность наблюдений, традиционные методы статистического анализа не проявляют свойства состоятельности.

Большой шаг вперед был сделан в результате работ [3]-[6], вьполненных по инициативе А. Н. Колмогорова, где был развит новый асимптотический подход, в котором рассматривается последовательность статистических задач растущей размерности с объемами выборок растушими так, что отношение размерности к объему выборки стремится к константе. Эта константа стала дополнительным параметром асимптотической теории. В противоположность традиционному асимптотическому подходу в математической статистике, новый подход получил название “асимптотики растущей размерности", а.p.p. (см. в [7; гл. 2]). Оказалось, что члены порядка величины отношения размерности к объему выборки описывают ряд сушественно многомерных явлений таких, как накопление погрешностей оценок, образование асимптотически неслучайных конечных смешений и множителей при убываюшей дисперсии и проявление эффектов, связанных с вырождением выборочных ковариационных матриц. Анализ ведущих членов этой асимптотики привел к развитию систематической теории существенно многомерного статистического анализа, главные достижения которой мы излагаем ниже (цитируя только основные публикации).

Раздел 1 посвяшен изложению первых результатов применения а.р.p., в основном, для изучения оценки качества стандартного линейного дискриминантного анализа нормальных совокупностей (А.Н. Колмогоров, Ю.Н.Благовешенский, А. Д. Деев, Л.В. Архаров, Л. Д. Мешалкин). В разделе 2 описьвается развитие предельной спектральной теории выборочных ковариационных матриц на базе методов предельной спектральной теории случайных матриш растущей размерности (В.А. Марченко и Л. А. Пастур, В. Л. Гирко и др.). Начиная с публикации [8] 1983 г. эта теория становится инструментальной базой для развития теории существенно многомерного статистического анализа. До последнего времени а.p.p. развивалась в форме предельных теорем и только иногда давались оценки скорости сходимости. В разделах 3-6 излагаются новые асимптотические исследования, отличающиеся тем, что главные члены а.p.p. выделяются при фиксированной размерности наблюдений и фиксированном объеме выборки; при этом остаточные члены оцениваются сверху (с точностью до абсолютных констант). Малость остаточных членов гарантируется при больших объемах выборок и большом числе ограниченно зависимых переменных. В разделе 3 развивается уточненная теория спектральных свойств выборочных ковариационных матриц. Для облегчения понимания сути и методов предлагаемого подхода основная теорема о связи между спектрами выборочных и истинных ковариационных матриц сначала излагается (в простейшей постановке) со всеми выкладками. В разделе 4 решается задача выделения в а.p.p. главных частей квадратичного риска обобщенных процедур линейной регрессии со случайными предикторами и откликом. В разделе 5 рассматривается расширенный класс линейных дискриминантных функций и с помощью методов а.p.p. решается задача оценки главной части вероятности ошибки. Раздел 6 носит итоговый характер: в нем показано, что в существенно многомерных задачах главные части традиционных функций качества регуляризованных многомерных статистических процедур зависят только от двух моментов наблюдений и могут быть 
вычислены (с точностью до остаточных членов а.р.p.) в предположении о нормальности наблюдений. В разделе 6 найдены в явном виде верхние оценки погрешности, которую дает предположение о нормальности. Теория, развитая в разделах $1-6$, позволяет для ряда регуляризованных вариантов многомерных статистических процедур и достаточно широкого класса совокупностей при высокой размерности наблюдений (1) установить слабую зависимость функционалов качества от распределений, (2) предложить надежные оценки качества этих процедур и, тем самым (3) обеспечить возможность сравнения процедур и отыскания приближенно неулучшаемых решений.

Введем основные обозначения. Пусть $\mathscr{S}$ всюду ниже обозначает $n$-мерную совокупность. Векторы х из $\mathscr{S}$ будем называть наблюдениями. Обозначим $\Sigma=\operatorname{cov}(\mathbf{x}, \mathbf{x})$. Будем рассматривать выборки $\mathscr{X}=\left(\mathbf{x}_{1}, \ldots, \mathbf{x}_{N}\right)$ из $\mathscr{S}$ объема $N$ и использовать векторы средних и матрицы

$$
\overline{\mathbf{x}}=N^{-1} \sum_{m=1}^{N} \mathbf{x}_{m}, \quad \mathbf{C}=N^{-1} \sum_{m=1}^{N}\left(\mathbf{x}_{m}-\overline{\mathbf{x}}\right)\left(\mathbf{x}_{m}-\overline{\mathbf{x}}\right)^{T}
$$

$$
\mathbf{S}=N^{-1} \sum_{m=1}^{N} \mathbf{x}_{m} \mathbf{x}_{m}^{T}
$$

(матрицы $\mathbf{S}$ могут иметь смысл выборочных ковариационных матриц, если ожидаемые значения $\mathbf{x}$ известны априори). Пусть везде ниже $\mathbf{E}$ означает оператор математического ожидания, a var - функцию дисперсии. Индикаторную функцию ind $(\mathscr{E})$, paвную 1 , если событие $\mathscr{E}$ имеет место, иначе 0 , будем применять также для неслучайных неравенств. Векторы будем выделять полужирным шрифтом, верхним индексом “ $T$ ” будем отмечать транспонированньй вектор-столбец. Абсолютная величина вектора означает его длину, а квадраты векторов суть квадраты их длин. Будем использовать только спектральные нормы матриц. Единичную матрицу обозначим буквой I.

\section{1. Метод растущей размерности в задачах многомерного статистического анализа}

Существенно многомерный подход к статистическим задачам был развит в 1968-1988 гг. сначала применительно к дискриминантному анализу. Опишем успехи, достигнутые к 1983 г. Поставим задачу дискриминантного анализа следуюшим образом.

Даны две различные совокупности $\mathscr{S}_{\nu}, \nu=1,2$, и по выборкам $\mathscr{X}_{\nu}=\left(\mathbf{x}_{1}, \ldots, \mathbf{x}_{N_{\nu}}\right)$ из $\mathscr{S}_{\nu}, \nu=1,2$, строится дискриминантная функция $w(\mathbf{x})=w\left(\mathbf{x}, \mathscr{X}_{1}, \mathscr{X}_{2}\right)$ и выбирается порог $c$. Правило дискриминации имеет вид $w(\mathbf{x})>c$ против $w(\mathbf{x}) \leqslant c$. Вероятности ошибок (условные при фиксированных выборках) суть

$$
\alpha_{1}=\mathbf{P}\left(w(\mathbf{x}) \leqslant c \mid \mathbf{x} \in \mathscr{S}_{1}\right), \quad \alpha_{2}=\mathbf{P}\left(w(\mathbf{x})>c \mid \mathbf{x} \in \mathscr{S}_{2}\right) .
$$

Для нормальных совокупностей $\mathscr{S}_{\nu}=\mathbf{N}\left(\mu_{\nu}, \Sigma\right), \nu=1,2$, с общей невырожденной известной ковариационной матрищей $\Sigma$ наименьшее значение $\left(\alpha_{1}+\alpha_{2}\right) / 2$ дает дискриминантная функция Андерсона

$$
w^{0}(\mathbf{x})=\left(\mu_{1}-\mu_{2}\right)^{T} \Sigma^{-1}\left(\mathbf{x}-\left(\mu_{1}+\mu_{2}\right) / 2\right) \quad \text { с порогом } c=0 .
$$


При этом $\alpha_{1}=\alpha_{2}=\Phi(-\sqrt{J} / 2)$, где $J=\left(\mu_{1}-\mu_{2}\right)^{T} \Sigma^{-1}\left(\mu_{1}-\mu_{2}\right)-$ "квадрат расстояния Махаланобиса". В стандартной процедуре дискриминантного анализа используется "подстановочная" дискриминантная функция Фишера-Андерсона-Вальда

$$
w(\mathbf{x})=\left(\overline{\mathbf{x}}_{1}-\overline{\mathbf{x}}_{2}\right)^{T} \mathbf{C}^{-1}\left(\mathbf{x}-\left(\overline{\mathbf{x}}_{1}+\overline{\mathbf{x}}_{2}\right) / 2\right),
$$

где $\overline{\mathbf{x}}_{1}$ и $\overline{\mathbf{x}}_{2}-$ векторы средних выборочных, а

$$
\mathbf{C}=(N-2)^{-1}\left[\sum_{m=1}^{N_{1}}\left(\mathbf{x}_{m}-\overline{\mathbf{x}}_{1}\right)\left(\mathbf{x}_{m}-\overline{\mathbf{x}}_{1}\right)^{T}+\sum_{m=N_{1}+1}^{N}\left(\mathbf{x}_{m}-\overline{\mathbf{x}}_{2}\right)\left(\mathbf{x}_{m}-\overline{\mathbf{x}}_{2}\right)\right]^{T}
$$

- несмещенная оценка $\Sigma$, в которой используется сквозная нумерация векторов выборок, причем сначала нумеруются векторы $\mathscr{X}_{1}$, а затем векторы $\mathscr{X}_{2}, N=N_{1}+N_{2}$. Вальд [9] доказал состоятельность этой процедуры при невырожденной матрище $\Sigma$ и $N_{1} \rightarrow \infty$ и $N_{2} \rightarrow \infty$.

В 1968 г. А. Н. Колмогоров в связи с несовершенством этой процедуры (матрица С может оказаться вырожденной, а при $n>N \mathbf{C}^{-1}$ заведомо не существует) обратил внимание на необходимость изучения зависимости вероятностей ошибок дискриминантного анализа от объемов выборок и решил следуюшую задачу. Пусть известно, что матрица $\Sigma$ единичная. Рассмотрим упрощенную подстановочную дискриминантную функцию $w^{1}(\mathbf{x})=\left(\overline{\mathbf{x}}_{1}-\overline{\mathbf{x}}_{2}\right)^{T}\left(\mathbf{x}-\left(\overline{\mathbf{x}}_{1}+\overline{\mathbf{x}}_{2}\right) / 2\right)$. Эта функция имеет нормальное распределение и приводит к вероятностям ошибок вида $\Phi\left(-G^{2} / D\right)$, где случайные $G$ и $D$ имеют нецентральное $\chi^{2}$-распределение. Чтобы выделить главные части $G$ и $D$, А.Н. Колмогоров предложил рассматривать не одну $n$-мерную задачу, а последовательность $\mathscr{P}=\left\{\mathscr{P}_{n}\right\}$ дискриминантных задач

$$
\mathscr{P}_{n}=\left(\mathscr{S}_{1}, \mathscr{S}_{2}, N_{1}, N_{2}, X_{1}, X_{2}, w(\mathbf{x}), \alpha_{1}, \alpha_{2}\right)_{n}, \quad n=1,2, \ldots
$$

(индексы $n$ у аргументов $\mathscr{P}_{n}$ выписывать не будем) анализа наблюдений $\mathbf{x} \in \mathbb{R}^{n}$, где по выборкам $\mathscr{X}_{1}$ и $\mathscr{X}_{2}$ объемов $N_{1}$ и $N_{2}$ из совокупностей $\mathscr{S}_{1}$ и $\mathscr{S}_{2}$ строится дискриминантная функция $w(\mathbf{x})$ и вычисляются вероятности ошибок $\alpha_{1}$ и $\alpha_{2}$. В предположении, что при каждом $n \mathscr{S}_{\nu}=\mathbf{N}\left(\mu_{\nu}, \mathbf{I}\right), \nu=1,2,\left(\mu_{1}-\mu_{2}\right)^{2} \rightarrow J_{0} \geqslant 0$ и $n / N_{\nu} \rightarrow \lambda_{\nu}>0$, $\nu=1,2$, для дискриминантной функции $w(\mathbf{x})=w^{1}(\mathbf{x})$ при $c=0$ он нашел, что по вероятности $\alpha_{1} \rightarrow \Phi\left(-J_{0} / 2 \sqrt{J_{0}+\lambda_{1}+\lambda_{2}}\right)$ (для $\alpha_{2}$ предел тот же).

В [6] Л. Д. Мешалкин вывел это же предельное выражение вероятностей ошибок дискриминантного анализа для совокупностей, отличных от нормальных, в предположении о сближении совокупностей в пространстве параметров и независимости компонент векторов наблюдений. В [10] этот результат был обобщен. В [11] показано, что то же предельное выражение для вероятностей ошибок остается справедливьм и тогда, когда используется обращение выборочной ковариационной матришы, но она имеет специальную структуру, и эта структура известна априори. В постановке [10] был вьполнен ряд работ по улучшению процедуры дискриминации сближающихся совокупностей с растущим числом блоков независимых компонент векторов наблюдений, см. [12], [14], [15]; в этих работах развита теория взвешивания и отбора вкладов независимых переменных в задаче дискриминантного анализа.

В 1970 г. Ю. Н. Благовешенский и А. Д. Деев исследовали в а.р.p. вероятности ошибок стандартной дискриминантной процедуры для двух нормальных совокупностей 
с совпадаюшими ковариационными матрицами. В [3] и [4] найдено асимптотическое разложение функции $\mathbf{E P}\left(w(\mathbf{x})<c, \mathbf{x} \in \mathscr{S}_{1}\right)$ для $w(\mathbf{x})$ вида $(1.2)$. Главньй результат состоит в следующем. Пусть в последовательности $\mathscr{P}=\left\{\mathscr{P}_{n}\right\}$ задач (1.4) по выборкам строится дискриминантная функция $w(\mathbf{x})$ и классификация вьполняется по правилу $w(\mathbf{x})>c$ против $w(\mathbf{x}) \leqslant c$.

ТЕОРемА 1.1 [3]. Пусть ЯР удовлетворяет следующим условиям.

(А) При каждом $n$ совокупности нормальнь $\mathbf{N}\left(\mu_{\nu}, \Sigma\right), \nu=1,2$, с общей невырожденной матрицей ковариации $\Sigma$.

(Б) При $n \rightarrow \infty$ существует предел $\lim \left(\mu_{1}-\mu_{2}\right)^{T} \Sigma^{-1}\left(\mu_{1}-\mu_{2}\right)=J$.

(B) При $n \rightarrow \infty, N_{\nu} \rightarrow \infty, \nu=1,2$, причем так, что $n / N_{\nu} \rightarrow \lambda_{\nu}>0, \nu=1,2$, $u \lambda \stackrel{\text { def }}{=} \lambda_{1} \lambda_{2} /\left(\lambda_{1}+\lambda_{2}\right)<1$.

Тогда по вероятности

$$
\alpha_{1} \rightarrow \Phi\left(-\sqrt{1-\lambda}\left(J-\lambda_{1}+\lambda_{2}-2 c\right) / 2 \sqrt{J+\lambda_{1}+\lambda_{2}}\right)
$$

(предельное значение для $\mathbf{E} \alpha_{2}$ записывается симметричным образом).

Легко убедиться, что минимум предельного значения $\left(\alpha_{1}+\alpha_{2}\right) / 2$ достигается при пороге $c=\left(\lambda_{1}-\lambda_{2}\right) / 2$, т.е. при классификации с предпочтением меньшей выборки. Стало очевидным, что учет членов порядка $n / N_{\nu}, \nu=1,2$, открывает путь для построения улучшенных дискриминантных (и других) процедур.

Для нормальных распределений матрица (1.3) есть матрища Уишарта, и для нее известны аналитические выражения как для плотности распределения элементов матрищы $\mathbf{C}$, так и для собственных значений [16], [17]. Однако использовать эти выражения в а.p.p. не удается. В [5] для матрищ Уишарта W с $\Sigma=\mathbf{I}$ в а.p.p. были найдены (путем явного вычисления) рекуррентные соотношения для предельных моментов $M_{k}=\operatorname{plim}_{n \rightarrow \infty} n^{-1} \operatorname{tr} \mathbf{W}^{k}, k=1,2, \ldots$, и доказано существование предельной функции распределения

$$
F(u)=\operatorname{plim}_{n \rightarrow \infty} n^{-1} \sum_{i=1}^{n} \operatorname{ind}\left(\lambda_{i} \leqslant u\right),
$$

где $\lambda_{1}, \ldots, \lambda_{n}$ - собственные значения W. В [5] была предпринята попытка численно восстановить $F(u)$ по моментам $\left\{M_{k}\right\}$. Между тем, эта функция была найдена аналитически ранее (как следствие из теоремы 1 из [18], см. ниже). Для $\mathbf{x} \sim \mathbf{N}(0, \Sigma)$ моменты $M_{k}$ в а.p.p. найдены (дифференцированием интегралов по параметрам) в [8]. При $y>0$ установлена формула $M_{k}=\left(\mathbf{L}^{k}\right)_{11} / y, k=1,2, \ldots$, где бесконечная матрища $\mathbf{L}$ состоит из элементов $\mathbf{L}_{i j}$, равных нулю при $j<i-1 ; 1$ при $j=i-1$; и $y \Lambda_{j-i+1}$ при $j>i-1$, для $i, j=1,2, \ldots$, где по определению $\Lambda_{k}=\lim _{n \rightarrow \infty} n^{-1} \operatorname{tr} \Sigma^{k}, k=1,2, \ldots$.

В [8] для нормальных $\mathbf{x} \sim \mathbf{N}(0, \Sigma)$ в а.p.p. найдена главная часть функции $\mathbf{E} n^{-1} \operatorname{tr}(\mathbf{I}+t \mathbf{C})^{-1}$, где матрица $\mathbf{C}$ вида (1.3) есть матрица Уишарта. Приведем главньй результат.

Пусть $\mathscr{P}=\left\{\mathscr{P}_{n}\right\}-$ последовательность статистических задач

$$
\mathscr{P}_{n}=(\mathscr{S}, \Sigma, N, \mathscr{X}, \mathbf{C})_{n}, \quad n=1,2, \ldots,
$$

в которых по выборкам $\mathscr{X}$ объемов $N$ из совокупностей $\mathscr{S}$ с $\operatorname{cov}(\mathbf{x}, \mathbf{x})=\Sigma$ строится выборочная ковариационная матрища $\mathbf{C}$ вида (1.3) и изучается ее спектр (индексы $n$ у аргументов $\mathscr{P}_{n}$ выпиисывать не будем). 
ТЕОремА 1.2 [8]. Пусть Я удовлетворяет следующим требованиям:

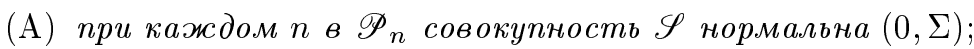

(Б) при каждом $n$ все собственные числа $\Sigma$ лежсат на отрезке $\left[c_{1}, c_{2}\right]$, где $c_{1}>0$ и $c_{2}$ от $n$ не зависят;

(В) $п р и$ любом $t \geqslant 0$ при $n \rightarrow \infty$ в Я имеет место сходимость $n^{-1} \operatorname{tr}(\mathbf{I}+t \Sigma)^{-1} \rightarrow \eta(t)$

(Г) существует предел $y=\lim _{n \rightarrow \infty} n / N>0$.

Тогда при любом $t \geqslant 0$ существует предел по вероятности

$$
h(t)=\operatorname{plim}_{n \rightarrow \infty} n^{-1} \operatorname{tr}(\mathbf{I}+t \mathbf{C})^{-1}
$$

и выполняется уравнение

$$
h(t)=\eta(t s(t)), \quad \text { əде } \quad s(t)=1-y+y h(t) .
$$

Значение уравнения (1.6) состоит в том, что оно позволяет связать предельные спектральные функции выборочных и неизвестных истинных ковариационных матрищ.

Заметим, что моменты $M_{k}$ могут быть вычислены дифференцированием функции $h(t)$. При $\Sigma=\mathbf{I}, n=1,2, \ldots$ уравнение (1.6) переходит в квадратное уравнение, найденное ранее в [18] (см. ниже, раздел 2).

\section{2. Предельная спектральная теория выборочных ковариационных матриц растущей размерности}

Эта теория представляет развитие теории случайных матриц, созданной сначала применительно к задачам теоретической физики. Изложим результаты, достигнутые в трудах В.А. Марченко и Л.А. Пастура, В. Л. Гирко и автора настоящей работы в 1967-1995 гг. В 1947 г. Е. Вигнер обнаружил сходимость спектральных функций случайных самосопряженных операторов в виде случайных матриц Грама растущей размерности вида $\mathbf{S}$ из (0.2) со свойствами выборочных ковариационных матриц для нормальных распределений и получил предельную спектральную плотность $f(u)$, пропорциональную $\sqrt{\left(u_{2}-u\right)\left(u-u_{1}\right)}, 0 \leqslant u_{1} \leqslant u_{2}$ ("полукруговой закон"). В 1967 г. В.А. Марченко и Л.А. Пастур в работе [18] исследовали предельные спектры самосопряженных операторов, представимых в виде суммы $\mathbf{A}+\mathbf{S}$, где $\mathbf{A}-$ неслучайные эрмитовы матрицы растущей размерности $n$ со сходящимися спектральными функциями, а $\mathbf{S}$ - случайные матрицы (0.2) при $\Sigma=\mathbf{I}$, и получили уравнение, связьвающее предельные спектральные функции матриц $\mathbf{A}$ и $\mathbf{A}+\mathbf{S}$. Приведем один из результатов этой работы.

Рассмотрим последовательность $\mathscr{P}=\left\{\mathscr{P}_{n}\right\}$ задач $\mathscr{P}_{n}=(\mathscr{S}, \Sigma, N, \mathscr{X}, \mathbf{S})_{n}$, $n=1,2, \ldots$, анализа спектров матрищ $\Sigma=\operatorname{cov}(\mathbf{x}, \mathbf{x})$ размера $n \times n$ по выборочным матрицам $\mathbf{S}$ вида (0.2), построенным по выборкам $\mathscr{X}$ из $\mathscr{S}$ объемов $N$ (индексы $n$ у матриц выписывать не будем). Обозначим

$$
h_{n}(t)=n^{-1} \operatorname{tr}(\mathbf{I}+t \mathbf{S})^{-1}, \quad F_{n}(u)=n^{-1} \sum_{i=1}^{n} \operatorname{ind}\left(\lambda_{i} \leqslant u\right),
$$

где $\lambda_{1}, \ldots, \lambda_{n}-$ собственные значения $\mathbf{S}$. 
ТЕОремА 2.1 (частный случай теоремы 1 из [18]). Пусть ЯР удовлетворяет следующим требованиям.

(А) При каждом $n$ для наблюдений $\mathbf{x}$ из $\mathscr{S}$ величина $\mathbf{E x}=0, \Sigma=\mathbf{I}$ и существуют все четвертые моменты компонент $\mathbf{x}$, равномерно ограниченные $\beta \mathscr{P}$.

(Б) Распределение $\mathbf{x}$ симметрично относительно перестановки компонент $\mathbf{x}$ и инвариантно относительно замены $\mathbf{x}$ на $-\mathbf{x}$. Это означает для компонент $\mathbf{x}=\left(x_{1}, \ldots, x_{n}\right)$, что

$$
\mathbf{E} x_{i} x_{j} x_{k} x_{l}=a_{n}\left(\delta_{i j} \delta_{k l}+\delta_{i k} \delta_{j l}+\delta_{i l} \delta_{j k}\right)+\left(b_{n}-3 a_{n}\right) \delta_{i j} \delta_{j k} \delta_{k l},
$$

$i, j, k, l=1, \ldots, n$ (здесь $\delta$ - символ Кронекера), $a_{n}=\mathbf{E} x_{1}^{2} x_{2}^{2}, b_{n}=\mathbf{E} x_{1}^{4}$.

(B) Существуют предельи $\lim _{n \rightarrow \infty} a_{n} u \lim _{n \rightarrow \infty} b_{n}$.

(Г) Существует предел $\lim _{n \rightarrow \infty} n / N=y>0$.

Тогда

$1^{\circ}$ при любом $t \geqslant 0$ существует предел $\operatorname{plim}_{n \rightarrow \infty} h_{n}(t)=h(t)$;

$2^{\circ}$ при $u \geqslant 0$ имеет место слабая сходимость $F_{n}(u) \stackrel{\mathbf{P}}{\longrightarrow} F(u)$;

$3^{\circ} n p u \operatorname{Re} z<0$ u npu $\operatorname{Im} z \neq 0 \quad h(z)=\int(1-z u)^{-1} d F(u)$;

$4^{\circ}$ справедливо равенство $h(t)(1+t s(t))=1$, где $s(t) \stackrel{\text { def }}{=} 1-y+y h(t), t \geqslant 0$.

ЗАмЕчАниЕ 1. Условия (Б) справедливы для изотропных распределений и, в частности, для нормальных распределений $\mathbf{N}(0, \mathbf{I})$. Предельная спектральная плотность матриц $\mathbf{S}$ в этом случае равна $d F(u) / d u=(2 \pi y u)^{-1} \sqrt{\left(u_{2}-u\right)\left(u-u_{1}\right)}$ при $u_{1} \leqslant u \leqslant u_{2}$, где $u_{1}=(1-\sqrt{y})^{2}, u_{2}=(1+\sqrt{y})^{2}$, и равна нулю при $0<u<u_{1}$ и при $u>u_{2}$; при $y>1$ функция $F(u)$ имеет скачок в точке $u=0$, равньй $1-y^{-1}$.

ЗАмЕчАниЕ 2 . Пусть векторы наблюдений нормальны $\left(0_{n}, \mathbf{I}_{n}\right), n=1,2, \ldots$ Тогда существует линейное преобразование переменных, преобразуюшее матришы С вида (0.1) к матрицам $\mathbf{S}$ вида (0.2) (с $N$ на единицу меньше) и теорема 1 из [18] справедлива также для матрищ $\mathbf{C}$ вида $(0.1)$.

Дальнейшие успехи применения а.p.p. в теории многомерного статистического анализа стали возможными благодаря применению метода спектральных функций, развитого в предельной спектральной теории случайных матрищ растущей размерности В. Л. Гирко (монографии [19] и [20]). В серии исследований [19]-[22] и др. были изучены предельные спектральные свойства матриц $\mathbf{S}$ вида (0.2). Для нормированного следа резольвенты матриц $\mathbf{S}$ была установлена сходимость почти наверное, установлена скорость сходимости, изучены предельные (полукруговые) спектры и доказано, что эти спектры остаются в финитных границах с вероятностью 1. Этот класс матриц нашел применение в некоторых задачах теоретической физики и в теории динамики нейронных сетей (А. Хорунжий, Г. Роджерс, А. Буте де Монвель, В. Василюк и др.). Заметим, что матрицы вида $\mathbf{S}$ могут иметь смысл выборочных ковариационных матриц в специальной постановке статистических задач, когда математические ожидания наблюдений известны априори (или равны 0 ).

Методы, развитые в [19]-[22], были применены к стандартным выборочным ковариационным матрицам С вида (0.1) в [23]-[29]. Использование метода спектральных 
функций для изучения спектров выборочных ковариационных матриц проиллюстрировано ниже при изложении теоремы 3.1. Сначала устанавливается убьвание дисперсии в а.p.p. для некоторых функционалов, зависящих от резольвенты матриц $\mathbf{S}$ или $\mathbf{C}$ и векторов $\overline{\mathbf{x}}$. Для этого применяются мартингальные леммы (см. в разделе 3 и в [20]). Будем использовать следующее простое утверждение.

ЛЕмма 2.1 (следствие из неравенства Буркхолдера, [30; гл. 7]). Пусть $f(\mathscr{X})-$ функиия въборки $\mathscr{X}=\left(\mathbf{x}_{1}, \ldots, \mathbf{x}_{N}\right)$, a $f^{m}(\mathscr{X})$ функиионально не зависит от $\mathbf{x}_{m}$, $m=1, \ldots, N$, и у этих функиий существуют первые два момента.

Tогдa var $f(\mathscr{X}) \leqslant \mathbf{E} \sum_{m=1}^{N}\left(f(\mathscr{X})-f^{m}(\mathscr{X})\right)^{2}$.

Далее, исключается один из независимых векторов, и математические ожидания функционалов, содержаших резольвенту, связьваются с математическими ожиданиями функционалов, зависящих от $\Sigma$, и так, как в теореме 3.1 , устанавливается основное спектральное уравнение, связьвающее предельные спектры матриц $\mathbf{S}$ или $\mathbf{C}$ и $\Sigma$. Переход к предельньм спектрам вьполняется с помощью утверждений типа следуюшей леммы (теорема 3.2.4 в [20]).

ЛЕмма 2.2 [20]. Рассмотрим последовательность неотрицательно определенных симметричных случайных матрич $\mathbf{S}_{n}$ вида $(0.2)$ и функиий $h_{n}(t)=$ $n^{-1} \operatorname{tr}\left(\mathbf{I}+t \mathbf{S}_{n}\right)^{-1}$ и $F_{n}(u)=n^{-1} \sum_{i=1}^{n} \operatorname{ind}\left(\lambda_{i} \leqslant u\right)$, әде $\lambda_{i}-$ собственные значения $\mathbf{S}_{n}, i=1, \ldots, n, n=1,2, \ldots$. Сходимость $h_{n}(t) \rightarrow h(t)$ nри каждом $t \geqslant 0$ по вероятности необходима и достаточна, для того чтобь

$1^{\circ}$ при $и>0$ имела место слабая сходимость по вероятности $F_{n}(u) \rightarrow F(u)$ $u$

$2^{\circ}$ вьполнялось уравнение $\int(1+t u)^{-1} d F(u)=h(t), t \geqslant 0$.

Изложим результат исследования предельных спектральных функций выборочных ковариационных матриц, полученньй в [23].

Пусть $\mathscr{P}=\left\{\mathscr{P}_{n}\right\}-$ последовательность статистических задач

$$
\mathscr{P}_{n}=(\mathscr{S}, \Sigma, N, \mathscr{X}, \mathbf{C})_{n}, \quad n=1,2, \ldots,
$$

анализа спектров матриц $\Sigma=\operatorname{cov}(\mathbf{x}, \mathbf{x})$ размера $n \times n$ по выборочным матрицам $\mathbf{C}$ вида (0.1), построенным по выборкам $\mathscr{X}$ объемов $N$ (индексы $n$ у аргументов $(2.1)$ вьпиисьвать не будем).

Теорема 2.2 [23]. Предположим, что ЯР удовлетворяет следующим требованиям (с учетом [28]; в [23] предположения сложнее).

(А) При каждом $n \mathbf{E x}=0$ и существуют все четвертые моменты проекиий $\mathbf{x}$ на любую неслучайную ось, равномерно ограниченные в Я્Р. При каждом $n$ все собственные числа матрии, $\Sigma$ лежат на отрезке $\left[c_{1}, c_{2}\right]$, әде величинь $0<c_{1} \leqslant c_{2}$ от $n$ не зависят.

(Б) Величиньц $\sup _{\|\Omega\|=1} \operatorname{var}\left(\mathbf{x}^{T} \Omega \mathbf{x} / n\right) \rightarrow 0$, где $\Omega$ - неслучайные симметричные неотрицательно определенные матрицы единичной (спектральной) нормы.

(B) $\lim _{n \rightarrow \infty} n / N=y>0$.

(Г) При $и \geqslant 0$ имеет место слабая сходимость

$$
n^{-1} \sum_{i=1}^{n} \operatorname{ind}\left(\lambda_{i} \leqslant u\right) \rightarrow F_{0}(u)
$$

равномерно по $и$, где $\lambda_{1}, \ldots, \lambda_{n}-$ собственные числа $\Sigma$. 
Тогда

$1^{\circ}$ при $t \geqslant 0$ равномерно в среднем квадратичном

$$
n^{-1} \operatorname{tr}(\mathbf{I}+t \mathbf{S})^{-1} \rightarrow h(t) \quad u \quad n^{-1} \operatorname{tr}(\mathbf{I}+t \mathbf{C})^{-1} \rightarrow h(t)
$$

$2^{\circ}$ при и $>0$ имеет место слабая сходимость

$$
n^{-1} \sum_{i=1}^{n} \operatorname{ind}\left(\lambda_{i} \leqslant u\right) \rightarrow F(u)
$$

равномерно по $и$, где $\lambda_{1}, \ldots, \lambda_{n}-$ собственные числа $\mathbf{S}$ или $\mathbf{C}$;

$3^{\circ}$ npu $t \geqslant 0$

$$
h(t)=\int(1+t u)^{-1} d F(u)=\int(1+t s(t) u)^{-1} d F_{0}(u),
$$

где $s(t)=1-y+y h(t)$;

$4^{\circ}$ аналитическое продолжение $h(z)$ функции $h(t)$ на плоскости комплексных z удовлетворяет условию Гёльдера

$$
\left|h(z)-h\left(z^{\prime}\right)\right|<c\left|z-z^{\prime}\right|^{\zeta}, \quad \text { əде } \quad c>0 \quad u \quad \zeta>0 ;
$$

$5^{\circ}$ для каждого и $>0$ при $\varepsilon \rightarrow+0$ существует предельная плотность

$$
\frac{d F(u)}{d u}=(\pi u)^{-1} \lim \operatorname{Im} h\left(-u^{-1}-i \varepsilon\right) ;
$$

$6^{\circ}$ при $0<u<c_{1}(1-\sqrt{y})^{2} u u>c_{2}(1+\sqrt{y})^{2}$ плотность $\frac{d F(u)}{d u}=0$;

$7^{\circ}$ при $y>1 F($ и) имеет скачок в точке $u=0$, равный $1-1 / y$.

Предельные спектральные уравнения для матриц $\mathbf{S}$ и $\mathbf{C}$ совпадают, однако скорость сходимости может быть разной; кроме того, отличаются главные части спектральных функций, зависяших от векторов $\overline{\mathbf{x}}$.

Уравнения типа (2.2), связываюшие предельные распределения спектров случайных матриц и спектров матриц их математических ожиданий, называются в [20] и в [26] "каноническими". Они демонстрируют фундаментальное свойство спектров случайных матриц растушей размерности: в а.p.p. их (гладкие) спектральные функции оказьваются нечувствительными к моментам выше вторых и, следовательно, могут быть вычислены в предположении о нормальности.

В работах В.Л. Гирко [24]-[26] методы спектральной теории случайных матрищ растушей размерности [19], [20] применяются для изучения предельных спектральных свойств стандартных выборочных ковариационных матриц С. Основные результаты получены в предположении о независимости компонент векторов наблюдений $\mathbf{x}$ (в монографии [26] 1995 г. это требование заменено требованием к параметрам). В работах [24]-[26] доказан ряд теорем, устанавливаюших сходимость спектральных функций матриц $\mathbf{C}$ в а.p.p., выведены уравнения связи меж ду предельными спектральными функциями матриц $\Sigma$ и $\mathbf{C}$, построены оценки нормированного следа резольвенты $\Sigma$, доказано, что эти оценки асимптотически нормальны, изучены гранипы предельных 
спектров выборочных ковариационных матриц. Приведем три утверждения, доказанные в [26] (1995 г.).

Пусть $\mathscr{P}=\left\{\mathscr{P}_{n}\right\}$ - последовательность статистических задач $(2.1)$ изучения спектральных функций матриц $\Sigma$ растушей размерности по матрицам С вида (0.1). Предположим следующее.

(А) При каждом $n$ в $\mathscr{P} \mathbf{E x}=0$, матрицы $\Sigma$ невырождены, а векторы $\xi=\Sigma^{-1 / 2} \mathbf{x}$ в $\mathscr{S}$ имеют независимые компоненты.

(Б1) Компоненты $\xi_{i}$ векторов $\xi$ удовлетворяют в $\mathscr{P}$ условию Линдеберга: для любого $\tau>0$ при $n \rightarrow \infty$ величина

$$
n^{-1} \sum_{i=1}^{n} \mathbf{E} \xi_{i}^{2} \text { ind }\left(\left|\xi_{i}\right|>\sqrt{n} \tau\right) \rightarrow 0 .
$$

(Б2) При каждом $n$ в $\mathscr{P}$ для всех компонент $\mathbf{x}$ сушествуют моменты порядка выше четвертого, равномерно ограниченные при $n=1,2, \ldots$, а собственные числа $\Sigma$ лежат на отрезке $\left[c_{1}, c_{2}\right]$, где $c_{1}>0$ и $c_{2}$ от $n$ не зависят.

(В) При $n \rightarrow \infty$ в $\mathscr{P}$ отношения $y_{n} \stackrel{\text { def }}{=} n / N \rightarrow y>0$.

Tеорема 2.3. Пусть в $\mathscr{P}=\left\{\mathscr{P}_{n}\right\}$ выполнены условия (A), (Б1) и (B). Тогда

$1^{\circ}$ при любом $n$ и любом $t \geqslant 0$ однозначно разрешима система уравнений

$$
h_{n}(t)=n^{-1} \operatorname{tr}\left(\mathbf{I}+t s_{n}(t) \Sigma\right)^{-1}, \quad s_{n}(t)=1-y_{n}+y_{n} h_{n}(t) ;
$$

$2^{\circ}$ при кажсдом $t \geqslant 0$ при $n \rightarrow \infty$ имеет место сходимость по вероятности $h_{n}(t)-\widehat{h}_{n}(t) \rightarrow 0$, zде $\widehat{h}_{n}(t)=n^{-1} \operatorname{tr}(\mathbf{I}+t \mathbf{C})^{-1}$

$3^{\circ}$ при и $>0$ имеет место сходимость по вероятности

$$
n^{-1} \sum_{i=1}^{n} \operatorname{ind}\left(\lambda_{i} \leqslant u\right)-F_{n}(u) \rightarrow 0,
$$

где $\lambda_{i}, i=1, \ldots, n,-$ собственнье числа $\mathbf{C}$, а функиия распределения $F_{n}(u)$ удовлетворяет уравнению

$$
\int(1+t u)^{-1} d F_{n}(u)=h_{n}(t), \quad t \geqslant 0
$$

TЕОРемА 2.4. Пусть в $\mathscr{P}=\left\{\mathscr{P}_{n}\right\}$ выполнень условия (A), (Б2) и (В).

Тогда при любом $t \geqslant 0$ надлежсащим способом иентрированная и нормированная величина $\widehat{h}_{n}(t)$ имеет функиию распределения, которая сходится при $n \rightarrow \infty \kappa$ функиии стандартного нормального закона.

Tеорема 2.5. Пусть в $\mathscr{P}=\left\{\mathscr{P}_{n}\right\}$ выполнены условия (A), (Б2) $и$ (B).

Тогда для минимального и максимального собственных чисел $\lambda_{1} u \lambda_{2}$ матрич $\mathbf{C}$ в $\mathscr{P}$ имеет место сходимость по вероятности $\lambda_{\nu}-\alpha_{\nu} \rightarrow 0, \nu=1,2$, где

$$
\alpha_{\nu}=z_{\nu}^{-1}\left[1+z_{\nu} N^{-1} \operatorname{tr} \Sigma\left(\mathbf{I}-z_{\nu} \Sigma\right)^{-1}\right], \quad \nu=1,2,
$$

a $z_{1}>0, z_{2}>0$ - минимальный и максимальный корни уравнения

$$
z^{2} N^{-1} \operatorname{tr} \Sigma^{2}(\mathbf{I}-z \Sigma)^{-2}=1 .
$$

В случае $\Sigma=\mathbf{I}$ отсюда следуют границы, указанные в замечании к теореме 2.1 . 


\section{3. Главные части резольвенты и спектральных функций выборочных ковариационных матриц}

В этом разделе мы перейдем, развивая результаты [27] и [28], от предельных формул спектральной теории выборочных ковариационных матриц [23]-[26], развитой к 1988 г., к соотношениям для главных частей спектральных функций, которые верны при любой фиксированной размерности наблюдений и любых фиксированных объемах выборки с оценкой остаточных членов сверху (с точностью до абсолютных констант). Мы покажем, что главные части спектральных функций выделяются с точностью до остаточных членов, величина которых определяется двумя параметрами, зависящими от первых четырех моментов наблюдений.

Известно, что предельные формулы теории спектральных свойств выборочных ковариационных матриц растущей размерности справедливы лишь при ограничениях зависимости между компонентами векторов наблюдений х. В работах [18] и [23] эти ограничения носят вид ограничений на тензорные выражения из моментов переменных. В основе теории В. Л. Гирко [19]-[20] лежит допущение о независимости компонент х. В работе автора [27] 1994 г. было обнаружено, что при $\mathbf{E x}=0$ условия ограниченной зависимости можно свести к ограничениям на два параметра: на максимальньй четвертьй момент проекции $\mathbf{x}$ на неслучайные оси (заданные векторами е единичной длины)

$$
M=\sup _{|\mathbf{e}|=1} \mathbf{E}\left(\mathbf{e}^{T} \mathbf{x}\right)^{4}
$$

и меру дисперсии квадратичных форм

$$
\nu=\sup _{\|\Omega\|=1} \operatorname{var}\left(\mathbf{x}^{T} \Omega \mathbf{x} / n\right)
$$

где $\Omega$ - неслучайные симметричные неотрицательно определенные матрищы единичной спектральной нормы. В [28] теория спектральных свойств выборочных ковариационных матриц развита для конечных $n$ и $N$. При $N \rightarrow \infty, M<\infty$ и $\nu \rightarrow 0$ неравенства работы [28] обеспечивают выполнение основных спектральных уравнений теории [19]-[26].

Метод выделения главньх членов а.p.p. Для иллюстрации основ излагаемой теории и ее математических приемов изложим существенно многомерный подход сначала со всеми выкладками на примере изучения (доказательства теоремы 3.1 , см. ниже), основной для [19]-[26] и [28] спектральной функции $h_{o}(t)=\mathbf{E} n^{-1} \mathbf{H}_{o}(t)$, где $\mathbf{H}_{o}(t)=(\mathbf{I}+t \mathbf{S})^{-1}$, для более простых матриц $\mathbf{S}$ вида $(0.2)$, построенных по выборкам $\mathscr{X}=\left\{x_{m}\right\}, m=1, \ldots, N$. Относительно совокупностей $\mathscr{S}$ предположим только, что $\mathbf{E x}=0$ и сушествуют все четвертые моменты компонент векторов х. Для простоты допустим, что $M>0$. Тогда можно обозначить $\gamma=\nu / M$. Обозначим, кроме того, $y=n / N, s_{o}(t)=1-y+y h_{o}(t)$ и $\tau=\sqrt{M} t$.

Так же, как в [22] и [28], воспользуемся методом попеременного исключения независимых векторов. Выделим вектор $\mathbf{x}_{m}$ из $\mathscr{X}$. Пусть по определению

$$
\begin{gathered}
\mathbf{S}^{m}=\mathbf{S}-N^{-1} \mathbf{x}_{m} \mathbf{x}_{m}^{T}, \quad \mathbf{H}_{o}^{m}=\left(\mathbf{I}+t \mathbf{S}^{m}\right)^{-1} \\
\varphi_{m}=\mathbf{x}_{m}^{T} \mathbf{H}_{o}^{m} \mathbf{x}_{m} / N, \quad \psi_{m}=\mathbf{x}_{m}^{T} \mathbf{H}_{o} \mathbf{x}_{m} / N
\end{gathered}
$$


где $m=1, \ldots, N$. Легко проверить тождества

$$
\begin{gathered}
\mathbf{H}_{o}=\mathbf{H}_{o}^{m}-t \mathbf{H}_{o}^{m} \mathbf{x}_{m} \mathbf{x}_{m}^{T} \mathbf{H}_{o} / N, \quad \mathbf{H}_{o} \mathbf{x}_{m}=\left(1-t \psi_{m}\right) \mathbf{H}_{o}^{m} \mathbf{x}_{m} \\
\left(1+t \varphi_{m}\right)\left(1-t \psi_{m}\right)=1
\end{gathered}
$$

$m=1, \ldots, N$. Очевидно,

$$
\left|\mathbf{e}^{T} \mathbf{H}_{o} \mathbf{x}_{m}\right| \leqslant\left|\mathbf{e}^{T} \mathbf{H}_{o}^{m} \mathbf{x}_{m}\right| \text { и } 0 \leqslant t \psi_{m} \leqslant 1, \quad m=1, \ldots, N .
$$

Убеждаемся также, что

$$
1-t \mathbf{E} \psi_{m}=1-t \mathbf{E} \operatorname{tr} \mathbf{H}_{o} \mathbf{S} / N=1-y \operatorname{tr}\left(\mathbf{I}-\mathbf{H}_{o}\right) / n=s_{o}(t),
$$

$m=1, \ldots, N$, и что $s_{o}(t) \geqslant(1+\sqrt{M} t y)^{-1}$.

ЛЕмма 3.1. При $t \geqslant 0$ дисперсия $\operatorname{var}\left(\mathbf{e}^{T} \mathbf{H}_{o} \mathbf{e}\right) \leqslant \tau^{2} / N$.

ДокАЗАТЕЛЬСтво. Оцениваем дисперсию с помошью леммы 2.1. Исключая зависимость от $\mathbf{x}_{m}$ согласно первому из тождеств (3.3), пользуемся (3.4) и находим, что

$$
\operatorname{var}\left(\mathbf{e}^{T} \mathbf{H}_{o} \mathbf{e}\right) \leqslant t^{2} N^{-2} \sum_{m=1}^{N} \mathbf{E}\left(\mathbf{e}^{T} \mathbf{H}_{o}^{m} \mathbf{x}_{m}\right)^{2}\left(\mathbf{x}_{m}^{T} \mathbf{H}_{o}^{m} \mathbf{e}\right)^{2} \leqslant t^{2} N^{-1} \mathbf{E}\left(\mathbf{e}^{T} \mathbf{H}_{o}^{m} \mathbf{x}_{m}\right)^{4} .
$$

В силу независимости $\mathbf{H}_{o}^{m}$ от $\mathbf{x}_{m}$ правая часть не больше $t^{2} N^{-1} M=\tau^{2} / N$. Лемма доказана.

Обозначим $\delta=2 \tau^{2} y^{2}\left(\gamma+\tau^{2} / N\right)$.

Лемма 3.2. При $t \geqslant 0 u N>1 \operatorname{var}\left(t \psi_{m}\right) \leqslant \delta, m=1, \ldots, N$.

ДокАЗАТЕЛЬСТвО. Выделяем один из векторов выборки $\mathbf{x}_{m}$ из $\mathbf{H}_{o}$ с помошью первого из тождеств (3.3). Обозначим $\Delta \varphi_{m}=\varphi_{m}-\mathbf{E} \varphi_{m}, \Delta \psi_{m}=\psi_{m}-\mathbf{E} \psi_{m}$. Из последнего из тождеств (3.3) видно, что

$$
\left(1+t \varphi_{m}\right) t \Delta \psi_{m}=\left(1-t \mathbf{E} \psi_{m}\right) t \Delta \varphi_{m}+t^{2} \mathbf{E} \Delta \varphi_{m} \Delta \psi_{m} .
$$

Возводим в квадрат и вычисляем ожидаемые значения. Получаем

$$
\operatorname{var}\left(t \psi_{m}\right) \leqslant \operatorname{var}\left(t \varphi_{m}\right)+\operatorname{var}\left(t \varphi_{m}\right) \operatorname{var}\left(t \psi_{m}\right)
$$

где $t \psi_{m} \leqslant 1$, и, значит, $\operatorname{var}\left(t \psi_{m}\right) \leqslant 2 \operatorname{var}\left(t \varphi_{m}\right)$. Чтобы применить лемму 2.1 , введем параметр $t^{\prime}=\left(1-N^{-1}\right) t$. Тогда $\mathbf{H}_{o}^{m}=\left(\mathbf{I}+t^{\prime} \mathbf{S}^{\prime}\right)^{-1}$, где $\mathbf{S}^{\prime}$ - матрица вида $\mathbf{S}$ для выборки на единицу меньшего объема $\left(\right.$ без $\left.\mathbf{x}_{m}\right)$. В силу независимости $\mathbf{S}^{\prime}$ и $\mathbf{x}_{m}$

$$
\operatorname{var}\left(t \varphi_{m}\right)=\operatorname{var}\left[t \mathbf{x}_{m}^{T}\left(\mathbf{E H}_{o}^{m}\right) \mathbf{x}_{m} / N\right]+t^{2} \mathbf{E}\left(\mathbf{x}_{m}^{2} / N\right)^{2} \operatorname{var}\left(\mathbf{e}^{T} \mathbf{H}_{o}^{m} \mathbf{e}\right),
$$

где $\mathbf{e}$ - единичный вектор в направлении $\mathbf{x}_{m}$, а вторая дисперсия в правой части условная при фиксированном е. Здесь первое слагаемое не больше $t^{2} y^{2} \nu=\tau^{2} \gamma$. Во втором слагаемом дисперсия в силу леммы 2.1 не больше $M t^{\prime 2} /(N-1) \leqslant M t^{2} / N=$ $\tau^{2} / N$, а $\mathbf{E}\left(\mathbf{x}_{m}^{2} / N\right)^{2} \leqslant M y^{2}$. Таким образом, второе слагаемое не больше $\tau^{4} y^{2} / N$. Тем самым лемма доказана. 
TeOPEMA 3.1. Прu $t \geqslant 0$

$$
h_{o}(t)=n^{-1} \operatorname{tr}\left(\mathbf{I}+t s_{o}(t) \Sigma\right)^{-1}+\omega,
$$

əде $|\omega| \leqslant \tau(\sqrt{\delta}+\tau / N)$.

ДокАЗАТЕЛЬСтво. Выберем какой-либо вектор $\mathbf{x}_{m} \in \mathscr{X}$. В силу (3.3)

$$
t \mathbf{H}_{o} \mathbf{x}_{m} \mathbf{x}_{m}^{T}=t\left(1-t \psi_{m}\right) \mathbf{H}_{o}^{m} \mathbf{x}_{m} \mathbf{x}_{m}^{T} .
$$

Здесь математическое ожидание левой части равно $t \mathbf{E H}_{o} \mathbf{S}=\mathbf{I}-\mathbf{E H}_{o}$. В правой части $1-t \psi_{m}=s_{o}(t)-\Delta_{m}$, где $\Delta_{m}$ - уклонение $t \psi_{m}$ от математического ожидания, а $\mathbf{E H}_{o}^{m} \mathbf{x}_{m} \mathbf{x}_{m}=\mathbf{E H}_{o}^{m} \Sigma$. Находим, что

$$
\mathbf{I}-\mathbf{E H}_{o}=t s_{o}(t) \mathbf{E H}_{o}^{m} \Sigma-t \mathbf{E} \mathbf{H}_{o}^{m} \mathbf{x}_{m} \mathbf{x}_{m}^{T} \Delta_{m} .
$$

Подставляем выражение для $\mathbf{H}_{o}^{m}$ через $\mathbf{H}_{o}$ из (3.3). Уравнение принимает вид

$$
\mathbf{I}=\mathbf{E H}_{o}\left(\mathbf{I}+t s_{o}(t) \Sigma\right)+\Omega,
$$

где $\Omega=t^{2} s(t) \mathbf{E} \mathbf{H}_{o}^{m} \mathbf{x}_{m} \mathbf{x}_{m}^{T} \mathbf{H}_{o} \Sigma / N-t \mathbf{E} \mathbf{H}_{o}^{m} \mathbf{x}_{m} \mathbf{x}_{m}^{T} \Delta_{m}$. Умножим слева на $\mathbf{R}=$ $\left(\mathbf{I}+t s_{o}(t) \Sigma\right)^{-1}$, вычисляем след и делим на $n$. Находим, что $n^{-1} \operatorname{tr} \mathbf{R}=h(t)+\omega$, где

$$
\omega=t^{2} s_{o}(t) \mathbf{E}\left|\mathbf{x}_{m}^{T} \mathbf{H}_{o} \Sigma \mathbf{R} \mathbf{H}_{o}^{m} \mathbf{x}_{m} / n\right| / N+t \mathbf{E}\left|\mathbf{x}_{m}^{T} \mathbf{R} \mathbf{H}_{o}^{m} \mathbf{x}_{m} \Delta_{m} / n\right| .
$$

В первом слагаемом $0 \leqslant s_{o}(t) \leqslant 1$. Оцениваем матричные выражения по норме, а во втором слагаемом применяем неравенство Шварца:

$$
|\omega| \leqslant t^{2}\|\Sigma\| \mathbf{E} \mathbf{x}_{m}^{2} / n N+t\left[\mathbf{E}\left(\mathbf{x}_{m}^{2} / n\right)^{2} \mathbf{E} \Delta_{m}^{2}\right]^{1 / 2} \leqslant \tau^{2} / N+\tau \sqrt{\operatorname{var} t \psi_{m}} .
$$

Отсюда следует утверждение теоремы.

Главные части (3.5) связывают спектры выборочных ковариационных матриц со спектрами истинных неизвестных ковариационных матриц и приводят к "каноническим" уравнениям предельной спектральной теории выборочных ковариационных матриц (см. [23]-[26]).

ПримеР. Пусть $\Sigma=\mathbf{I}$. Главная часть (3.5) дает квадратное уравнение $h_{o}(t)\left(1+t s_{o}(t)\right)=1$ относительно $h_{o}(t)=\mathbf{E} n^{-1} \operatorname{tr}(\mathbf{I}+t \mathbf{S})^{-1}$. Пользуясь аналитическим продолжением функции $h_{o}(t)$, можно вычислить спектральную функцию $F(u)=n^{-1} \sum \mathbf{P}\left(\lambda_{i} \leqslant u\right)$, где сумма вычисляется по всем собственным числам $\lambda_{i}$, $i=1, \ldots, n$, матришы $\mathbf{S}$. В предельной форме уравнение (3.5) впервые было получено в [18] (см. раздел 2).

Условия слабой зависимости переменных. Отметим, что ограниченность моментов $M$ существенно ограничивает зависимость переменных. Действительно, пусть $\Sigma$ - матрица корреляций с байесовьм распределением коэффициентов корреляции, равномерным от -1 до 1 . Тогда байесово среднее $\mathbf{E} M \geqslant \mathbf{E} \operatorname{tr} \Sigma^{2} \geqslant(n+2) / 3$. В случае $N(0, \Sigma)$ с $\Sigma$ из единиц величина $M=3 n^{2}$. Покажем, что уравнение (3.5) может быть установлено с точностью до членов, где моменты ограничены лишь в совокупности.

Обозначим

$$
\Lambda_{k}=n^{-1} \operatorname{tr} \Sigma^{k}, \quad Q_{k}=\mathbf{E}\left(\mathbf{x}^{2} / n\right)^{k}, \quad W=n^{-2} \sup _{\|\Omega\|=1} \mathbf{E}\left(\mathbf{x}^{T} \Omega \mathbf{x}^{\prime}\right)^{4},
$$

где $t, k \geqslant 0, \mathbf{x}$ и $\mathbf{x}^{\prime}-$ независимые векторы из $\mathscr{S}, \Omega$ - неслучайные симметричные неотрицательно определенные матрицы единичной спектральной нормы. 
TeOpema 3.2. Прu $t \geqslant 0$

$$
h_{o}(t)=n^{-1} \operatorname{tr}\left(\mathbf{I}+t s_{0}(t) \Sigma\right)^{-1}+\omega,
$$

где $\omega^{2} / 2 \leqslant\left[Q_{2} y^{2}\left(\nu+W t^{2} / N\right)+W / N^{2}\right] t^{4}$.

ПримеР. Пусть $\mathbf{x} \sim \mathbf{N}(0, \Sigma)$. Обозначим $\Lambda_{k}=n^{-1} \operatorname{tr} \Sigma^{k}, k=1,2, \ldots$ Для нормальных $\mathbf{x}$

$$
M=3\|\Sigma\|^{2}, \quad Q_{2}=\Lambda_{1}^{2}+2 \Lambda_{2} / n, \quad W=3\left(\Lambda_{2}^{2}+2 \Lambda_{4} / n\right), \quad \nu=2 \Lambda_{2} / n .
$$

Пусть теперь $\Sigma=\mathbf{I}+\rho \mathbf{E}$, где $\mathbf{E}$ - матрица из единиц, $0 \leqslant \rho \leqslant 1$. Тогда $M=3(1+n \rho)^{2}$, $\Lambda_{1}=1+\rho, \Lambda_{2}=1+2 \rho+n \rho^{2}, \Lambda_{k} \leqslant a_{k}+b_{k} \rho^{k} n^{k-1}$, где $a_{k}$ и $b_{k}$ - положительные числа, не зависяшие от $n$, и все $Q_{k}<c, k=1,2, \ldots$ При $\rho=n^{-3 / 4}$ и $n \rightarrow \infty$ величины $M \rightarrow \infty$, в то время как $\Lambda_{3}, \Lambda_{4}$ и $Q_{3}$ остаются ограниченными, $\nu=O\left(n^{-1}\right)$ и обеспечивается условие $\omega \rightarrow 0$.

Основные результаты. Приведем некоторые утверждения, доказанные в [27] и [28]. В этих работах выделены главные части не только функции $h_{o}(t)=n^{-1} \operatorname{tr} \mathbf{H}_{o}$, но и отдельных матричных элементов резольвент $\mathbf{H}_{o}$ и $\mathbf{H}$ матриц $\mathbf{S}$ и $\mathbf{C}$.

Для сокрашения записи оценок остаточных членов обозначим

$$
\varepsilon=\sqrt{\gamma+1 / N}, \quad c_{l m}=c_{l m}(t)=a \max \left(1, \tau^{l}\right) \max \left(1, \lambda^{m}\right),
$$

$l, m=1, \ldots, 9$, где $a$ - абсолютные константы (для краткости скобки у $c_{l m}(t)$ с зависимостью от $t$ мы вьписывать не будем).

TeOpema 3.3. Прu $t \geqslant 0$

$$
\mathbf{E H}_{o}=\left(\mathbf{I}+t s_{o}(t) \Sigma\right)^{-1}+\Omega_{o},
$$

әде $\left\|\Omega_{o}\right\|^{2} \leqslant c_{62} \varepsilon^{2}, u \operatorname{var} \mathbf{e}^{T} \mathbf{H}_{o} \mathbf{e} \leqslant \tau^{2} / N$.

В [28] показано, что отличие резольвент $\mathbf{H}_{o}$ и $\mathbf{H}$ сказывается только на остаточных членах. Получаем следуюшее утверждение.

TeOpema 3.4. Прu $t \geqslant 0$

$$
\mathbf{E H}=(\mathbf{I}+t s(t) \Sigma)^{-1}+\Omega,
$$

əде $\|\Omega\|^{2} \leqslant c_{63} \varepsilon^{2}, u \operatorname{var} \mathbf{e}^{T} \mathbf{H e} \leqslant a \tau^{2} / N$, әде а - абсолютная константа.

В качестве оценок функций $h_{o}(t), s_{o}(t)$ и $h(t)$ можно предложить статистики $\widehat{h}_{o}(t)=n^{-1} \operatorname{tr}(\mathbf{I}+t \mathbf{S})^{-1}, \widehat{s}_{o}(t)=1-y+y \widehat{h}_{o}(t)$, и $\widehat{h}(t)=n^{-1} \operatorname{tr}(\mathbf{I}+t \mathbf{C})^{-1}$.

TeOPEMA 3.5. Прu $t \geqslant 0$

(1) $\mathbf{E}\left|\widehat{h}_{o}(t)+h_{o}(t)\right|^{2} \leqslant \tau /(n N)$;

(2) $\mathbf{E}\left|\widehat{s}_{o}(t)-s_{o}(t)\right|^{2} \leqslant c_{12} /(n N)$;

(3) $\mathbf{E}|\widehat{h}(t)-h(t)|^{2} \leqslant c_{20} \varepsilon^{2}$.

Для оценки величин $s_{o}(t)$ можно предложить также статистику $\Psi(t)=\overline{\mathbf{x}}^{T} \mathbf{H}(t) \overline{\mathbf{x}}$ : 
ТЕОРемА 3.6. При $t \geqslant 0$ статистика $\tilde{s}_{o}(t)=(1+t \Psi(t))^{-1}$ моэсет служить оценкой $s_{\text {o }}(t)$ такой, что

$$
\mathbf{E} \tilde{s}_{o}(t)=s_{o}(t)+o, \quad \text { əde } \quad|o| \leqslant c_{42} \varepsilon, \quad u \quad \operatorname{var} \tilde{s}_{o}(t) \leqslant c_{64} / N
$$

В теории сушественно многомерного статистического анализа центральное место занимает теорема 3.4. С ее помощью можно получить и усилить теоремы о "канонических" уравнениях теории В. Л. Гирко [24]-[26] о связи между спектрами истинных и выборочных ковариационных матрищ (устремляя $\gamma$ к нулю), приближенно вычислить собственные числа матриц $\mathbf{C}$ и построить " $G$-оценки” спектральных функций матриц $\Sigma$ с гарантированным уклонением. Из нее следуют главные результаты [8] и [27]-[29]. Значение теоремы 3.4 состоит в том, что она представляет базис для построения улучшенных методов многомерного статистического анализа (см. разделы 4,5 и 6 ).

\section{4. Уменьшение квадратичного риска линейной регрессии с большим числом случайных предикторов}

В этом разделе мы изложим некоторые новые результаты, полученные применением методов, развитых в разделах 2 и 3 :

$1^{\circ}$ рассматривается класс обобщенных регуляризованных регрессионных процедур, зависящих от произвольной функции;

$2^{\circ}$ в а.p.p. выделяется главная часть квадратичного риска для этого класса регрессий и конструируется ее оценка;

$3^{\circ}$ погрешности, вызванные применением а.p.p., оцениваются сверху.

Пусть дана $(n+1)$-мерная совокупность $\mathscr{S}$, в которой векторы наблюдений есть пары $(\mathbf{x}, y)$, где $\mathbf{x}=\left(x_{1}, \ldots, x_{n}\right)$ рассматривается как вектор предикторов и скаляр $y$ как отклик.

Относительно совокупности $\mathscr{S}$ предположим только, что $\mathbf{E} x=0, \mathbf{E} y=0$, существуют все четвертые моменты компонент $\mathbf{x}$, четвертый момент $y$ и все четвертые моменты их произведений. Пусть $\mathbf{E x}^{2}>0$ (невырожденньй случай). Обозначим в этом разделе $M_{4}=\sup _{\mathbf{e}} \mathbf{E}\left(\mathbf{e}^{T} \mathbf{x}\right)^{4}, M_{8}=\mathbf{E}\left(\mathbf{x}^{2} / n\right)^{2} y^{4}$ и введем два параметра

$$
\begin{aligned}
M & =\max \left(M_{4}, \sqrt{M_{8}}, \mathbf{E} y^{4}\right), \\
\gamma & =\sup _{\Omega} \operatorname{var}\left(\mathbf{x}^{T} \Omega \mathbf{x} / n\right) M,
\end{aligned}
$$

где (и ниже) $\mathbf{e}$ - неслучайные векторы единичной длины, а $\Omega$ - симметричные неотрицательно определенные матрищы со спектральной нормой единища. Рассмотрим линейную регрессию $y=\mathbf{k}^{T} \mathbf{x}+l+\Delta$, где $\mathbf{k} \in \mathbb{R}^{n}$ и $l \in \mathbb{R}^{1}$. Требуется минимизировать функцию квадратичного риска $R=\mathbf{E} \Delta^{2}$ посредством наилучшего выбора оценок $\mathbf{k}$ и $l$ по выборке $\left\{\left(\mathbf{x}_{m}, y_{m}\right), m=1, \ldots, N\right\}$ из $\mathscr{S}$.

Обозначим $\lambda=n / N, \mathbf{a}=\mathbf{E x}, a_{0}=\mathbf{E} y, \Sigma=\operatorname{cov}(\mathbf{x}, \mathbf{x}), \sigma^{2}=\operatorname{var} y, \mathbf{g}=\operatorname{cov}(\mathbf{x}, y)$.

Если $\sigma>0$, а матрица $\Sigma$ невырожденная, то априорные коэффициенты $\mathbf{k}=\Sigma^{-1} \mathbf{g}$ и $l=a_{0}-\mathbf{k}^{T} \mathbf{a}$ доставляют минимум величине $R$, равньй $R=R^{o}=\sigma^{2}-\mathbf{g}^{T} \Sigma^{-1} \mathbf{g}=$ $\sigma^{2}\left(1-r^{2}\right)$, где $r$ есть коэффициент множественной корреляции. 
Исходим из статистик

$$
\begin{aligned}
& \overline{\mathbf{x}}=N^{-1} \sum_{m=1}^{N} \mathbf{x}_{m} \\
& \bar{y}=\sum_{m=1}^{N} y_{m} \\
& \widehat{\sigma}^{2}=N^{-1} \sum_{m=1}^{N}\left(y_{m}-\bar{y}\right)^{2} \\
& S=N^{-1} \sum_{m=1}^{N} \mathbf{x}_{m} \mathbf{x}_{m}^{T} \\
& \mathbf{C}=N^{-1} \sum_{m=1}^{N}\left(\mathbf{x}_{m}-\overline{\mathbf{x}}\right)\left(\mathbf{x}_{m}-\overline{\mathbf{x}}\right)^{T} \text { и } \\
& \widehat{g}=N^{-1} \sum_{m=1}^{N}\left(\mathbf{x}_{m}-\overline{\mathbf{x}}\right)\left(y_{m}-\bar{y}\right) .
\end{aligned}
$$

Стандартная "подстановочная" процедура с $\widehat{\mathbf{k}}=\mathbf{C}^{-1} \widehat{\mathbf{g}}$ и $\widehat{l}=\bar{y}-\widehat{\mathbf{k}}^{T} \overline{\mathbf{x}}$ обладает известными недостатками: эта процедура не гарантирует минимального риска, вырождается в случае мультиколлинеарных данных (когда вырождается матрица С) и не является состоятельной равномерно по числу измерений [31].

Квадратичный риск выборочной регрессии $y=\widehat{\mathbf{k}}^{T} \mathbf{x}+\widehat{l}+\Delta$, где $\widehat{\mathbf{k}}$ и $\widehat{l}$ рассчитьваются по выборкам, при “подстановочном" свободном члене $\widehat{l}=\bar{y}-\widehat{\mathbf{k}}^{T} \overline{\mathbf{x}}$ составляет

$$
R=\mathbf{E} \Delta^{2}=R^{1}+\mathbf{E}\left(\stackrel{\circ}{y}-\widehat{\mathbf{k}}^{T} \stackrel{\circ}{\mathbf{x}}\right)^{2}=(1+1 / N) R^{1},
$$

где

$$
R^{1 \stackrel{\text { def }}{=}} \mathbf{E}\left(\sigma^{2}-2 \widehat{\mathbf{k}}^{T} \mathbf{g}+\widehat{\mathbf{k}}^{T} \Sigma \widehat{\mathbf{k}}\right),
$$

$\stackrel{\circ}{y}=y-a_{0}$ и $\stackrel{\circ}{\mathbf{x}}=\overline{\mathbf{x}}-\mathbf{a}$. Поставим задачу вычисления и минимизации $R^{1}$.

Рассмотрим следуюший класс обобшенных регуляризованных регрессий. Пусть $\mathbf{H}_{o}=(\mathbf{I}+t \mathbf{S})^{-1}$ и $\mathbf{H}=(\mathbf{I}+t \mathbf{C})^{-1}-$ резольвенты матриш $\mathbf{S}$ и $\mathbf{C}$, где (как и вьше) $\mathbf{I}$ - единичная матрища. Коэффищиенты $\widehat{\mathbf{k}}$ будем выбирать (везде ниже) из класса $\mathscr{K}$ статистик вида $\widehat{\mathbf{k}}=\Gamma \widehat{\mathbf{g}}$, где матришы $\Gamma=\int t \mathbf{H}(t) d \eta(t)$, а $\eta(t)$ - функции с изменением на $[0, \infty)$, не большим 1, с достаточным числом моментов

$$
\eta_{k} \stackrel{\text { def }}{=} \int t^{k}|d \eta(t)|, \quad k=1,2, \ldots
$$

Отметим, что функция $\eta(t)$ вида единичной ступеньки соответствует "гребневой регрессии", см. [31]. Уравнение регрессии с коэффициентами $\widehat{\mathbf{k}} \in \mathscr{K}$ можно назвать обобщенной гребневой регрессией. Величина (4.4) зависит от $\eta(t): R^{1}=R^{1}(\eta)$ и при $\widehat{\mathbf{k}} \in \mathscr{K}$

$$
R^{1}(\eta)=\sigma^{2}-2 \mathbf{E} \int t \mathbf{g}^{T} \mathbf{H}(t) \widehat{\mathbf{g}} d \eta(t)+\mathbf{E} \iint D(t, u) d \eta(t) d \eta(u)
$$

где

$$
D(t, u) \stackrel{\text { def }}{=} t u \widehat{\mathbf{g}}^{T} \mathbf{H}(t) \Sigma \mathbf{H}(u) \widehat{\mathbf{g}} .
$$

Поскольку в $R^{1}(\eta)$ все аргументы инвариантны относительно сдвига начала координат, принимаем, что $\mathbf{a}=\mathbf{E x}=0$ и $a_{0}=\mathbf{E} y=0$. Так же, как в [28], будем выделять 
главные части функционалов в а.p.p. и оценивать остаточные члены сверху с точностью до абсолютных констант. Для сокращения записи оценок остаточных членов будем использовать обозначения

$$
\begin{gathered}
\tau=\sqrt{M} t, \quad \varepsilon=\sqrt{\gamma+1 / N}, \\
c_{l m}=c_{l m}(t)=\alpha \max \left(1, \tau^{l}\right) \max \left(1, \lambda^{m}\right), \quad \alpha, l, m \geqslant 0,
\end{gathered}
$$

где $\alpha, l$ и $m$ - числа (для краткости скобки у $c_{l m}(t)$ с зависимостью от $t$ мы выписьвать не будем). Легко убедиться, что

$$
\begin{gathered}
\mathbf{E}\left(\mathbf{x}^{2}\right)^{2} \leqslant M, \quad \mathbf{E}\left(\overline{\mathbf{x}}^{2}\right)^{2} \leqslant M \lambda^{2}, \quad\|\Sigma\|^{2} \leqslant M, \quad \mathbf{g}^{2} \leqslant M \\
\mathbf{E}\left(\widehat{\mathbf{g}}^{2}\right)^{2} \leqslant 2 M^{2}\left(1+\lambda^{2}\right), \quad \mathbf{E}\left(\overline{\mathbf{x}}^{T} \widehat{\mathbf{g}}\right)^{2} \leqslant 2 M^{3 / 2}\left(1+\lambda^{2}\right) .
\end{gathered}
$$

Так же, как в разделе 3 , изучим сначала свойства функций матришы Грама $\mathbf{S}$ и ее резольвенты $\mathbf{H}_{o}$. Рассмотрим функции

$$
h_{o}(t)=n^{-1} \operatorname{tr} \mathbf{H}_{o}(t) \text { и } s_{o}(t)=1-\lambda+\lambda h_{o}(t) .
$$

Эти функции обладают свойствами

$$
1-s_{o}(t)=t N^{-1} \mathbf{E} \operatorname{tr} \mathbf{H}_{o}(t) \mathbf{S} \text { и }(1+\tau \lambda)^{-1} \leqslant s_{o}(t) \leqslant 1 .
$$

В силу теоремы 3.3

$$
\mathbf{E H}_{o}(t)=\left(\mathbf{I}+t s_{o}(t) \Sigma\right)^{-1}+\Omega_{o},
$$

где $\left\|\Omega_{o}\right\| \leqslant c_{31} \varepsilon$ и $\operatorname{var}\left(\mathbf{e}^{T} \mathbf{H}_{o}(t) \mathbf{e}\right) \leqslant \tau^{2} / N$.

$\mathrm{C}$ помошью метода исключения переменных выделим главные части некоторых функционалов, которые нам понадобятся ниже.

ЛЕмма 4.1. При $t \geqslant 0$

$1^{\circ}\left|t \mathbf{E} \overline{\mathbf{x}}^{T} \mathbf{H}_{o}(t) \widehat{\mathbf{g}}\right| \leqslant M^{1 / 4} c_{32} \varepsilon ;$

$2^{\circ} \operatorname{var}\left(t \overline{\mathbf{x}}^{T} \mathbf{H}_{o}(t) \widehat{\mathbf{g}}\right) \leqslant \sqrt{M} c_{42} / N$

ЛЕмма 4.2. При $t \geqslant 0$

$1^{\circ} t \mathbf{E} \mathbf{e}^{T} \mathbf{H}_{o}(t) \widehat{\mathbf{g}}=t s_{o}(t) \mathbf{E} \mathbf{e}^{T} \mathbf{H}_{o}(t) \mathbf{g}+o, 2 \partial e|o| \leqslant c_{31} \varepsilon ;$

$2^{\circ} t \mathbf{E}^{T} \mathbf{H}_{o}(t) \widehat{\mathbf{g}}=\sigma^{2}\left(1-s_{o}(t)\right)+t s_{o}(t) \mathbf{E g}^{T} \mathbf{H}_{o}(t) \widehat{\mathbf{g}}+o_{1}$ $=\sigma^{2}\left(1-s_{o}(t)\right)+t s_{o}^{2}(t) \mathbf{E g}^{T} \mathbf{H}_{o}(t) \mathbf{g}+o_{2}$, , əəe $\left|o_{1}\right| \leqslant \sqrt{M} c_{32} \varepsilon u\left|o_{2}\right| \leqslant \sqrt{M} c_{32} \varepsilon$.

ЛЕмма 4.3. При $t \geqslant u \geqslant 0$

$$
\begin{aligned}
t u \mathbf{E} \widehat{\mathbf{g}}^{T} \mathbf{H}_{o}(t) \mathbf{S} \mathbf{H}_{o}(u) \widehat{\mathbf{g}}=t & s_{o}(t) u s_{o}(u) \mathbf{E} \widehat{\mathbf{g}}^{T} \mathbf{H}_{o}(t) \Sigma \mathbf{H}_{o}(u) \widehat{\mathbf{g}} \\
& +\left(1-s_{o}(u)\right) t \mathbf{E} \widehat{\mathbf{g}}^{T} \mathbf{H}_{o}(t) \widehat{\mathbf{g}}+\left(1-s_{o}(t)\right) u \mathbf{E} \widehat{\mathbf{g}}^{T} \mathbf{H}_{o}(u) \widehat{\mathbf{g}} \\
& +\sigma^{2}\left(1-s_{o}(t)\right)\left(1-s_{o}(u)\right)+o,
\end{aligned}
$$

əəe $|o| \leqslant \sqrt{M} c_{42} \varepsilon$.

Для перехода к $\mathbf{C}, \mathbf{H}$ и $\widehat{\mathbf{g}}$ воспользуемся тождествами $\mathbf{C}=\mathbf{S}-\overline{\mathbf{x x}}^{T}$ и $\mathbf{H}(t)=\mathbf{H}_{o}(t)+$ $t \mathbf{H}_{o}(t) \overline{\mathbf{x x}}^{T} \mathbf{H}(t)$. Обозначим $U(t)=\mathbf{e}^{T} \mathbf{H}(t) \overline{\mathbf{x}}, \Psi(t)=\overline{\mathbf{x}}^{T} \mathbf{H}(t) \overline{\mathbf{x}}$. 
ЛЕмМА 4.4.

$1^{\circ} U(t)=V(t)+t U(t) \Phi(t),(1+t \Psi(t))(1-t \Phi(t))=1 ;$

$2^{\circ} t s_{o}^{2}(t)(\mathbf{E} U(t))^{2} \leqslant c_{63} \varepsilon^{2} ;$

$3^{\circ} t s_{o}(t) \Psi(t)=1-s_{o}(t)+o, 2 \partial e o^{2} \leqslant c_{74} \varepsilon^{2}$.

Обозначим

$$
\widehat{h}(t)=n^{-1} \operatorname{tr} \mathbf{H}(t), \quad h(t)=\mathbf{E} \widehat{h}(t), \quad s(t)=1-\lambda+\lambda h(t) .
$$

Нетрудно убедиться, что при $t \geqslant 0 \quad 0<s(t) \leqslant(1+\tau \lambda)^{-1}$.

ЛЕмма 4.5.

$1^{\circ}\left\|\mathbf{E H}(t)-\mathbf{E H}_{o}(t)\right\| \leqslant \min \left(c_{74} \varepsilon^{2}, c_{32} \varepsilon\right) ;$

$2^{\circ}\left|s(t)-s_{o}(t)\right| \leqslant c_{11} / N$.

Обозначим

$$
\phi(t)=t \mathbf{g}^{T}(\mathbf{I}+t \Sigma)^{-1} \mathbf{g}, \quad \kappa(t)=s(t) \phi(t s(t))+\sigma^{2}(1-s(t)) .
$$

ЛЕмма 4.6 .

$1^{\circ} t \mathbf{E} \mathbf{g}^{T} \mathbf{H}(t) \widehat{\mathbf{g}}=t s(t) \mathbf{E g}^{T} \mathbf{H}(t) \mathbf{g}+o, 2 \partial e|o| \leqslant \sqrt{M} c_{42} \varepsilon ;$

$2^{\circ} t \mathbf{E} \widehat{\mathbf{g}}^{T} \mathbf{H}(t) \widehat{\mathbf{g}}=\sigma^{2}(1-s(t))+t s(t) \mathbf{E g}^{T} \mathbf{H}(t) \widehat{\mathbf{g}}+o_{1}=\kappa(t)=o_{2}$, где $\left|o_{1}\right| \leqslant \sqrt{M} c_{42} \varepsilon u\left|o_{2}\right| \leqslant \sqrt{M} c_{42} \varepsilon$.

ЛЕмма 4.7. При $t \geqslant u \geqslant 0$

$1^{\circ} t u\left|\mathbf{E} \widehat{\mathbf{g}}^{T} \mathbf{H}(t) \Sigma \mathbf{H}(u) \widehat{\mathbf{g}}-\mathbf{E} \widehat{\mathbf{g}}^{T} \mathbf{H}_{o}(t) \Sigma \mathbf{H}_{o}(u) \widehat{\mathbf{g}}\right| \leqslant \sqrt{M} c_{63} \varepsilon ;$

$2^{\circ} t u\left|\mathbf{E} \widehat{\mathbf{g}}^{T} \mathbf{H}(t) \mathbf{C H}(u) \widehat{\mathbf{g}}-\mathbf{E} \widehat{\mathbf{g}}^{T} \mathbf{H}_{o}(t) \mathbf{S} \mathbf{H}_{o}(u) \widehat{\mathbf{g}}\right| \leqslant \sqrt{M} c_{43} \varepsilon$.

TEOPEMA 4.1. Прu $t \geqslant u \geqslant 0$

$$
\begin{aligned}
t u\left|\mathbf{E} \widehat{\mathbf{g}}^{T} \mathbf{H}(t) \mathbf{C H}(u) \widehat{\mathbf{g}}\right|=t u s(t) s(u) D(t, u)+(1-s(u)) t \mathbf{E} \widehat{\mathbf{g}}^{T} \mathbf{H}(t) \widehat{\mathbf{g}} \\
+(1-s(t)) u \mathbf{E} \widehat{\mathbf{g}}^{T} \mathbf{H}(u) \widehat{\mathbf{g}}+\sigma^{2}(1-s(t))(1-s(u))+o,
\end{aligned}
$$

где $D(t, u)$ определено в (4.6), а $\mathbf{E}|o| \leqslant \sqrt{M} c_{63} \varepsilon$.

Выразим сначала главную часть квадратичного риска $R^{1}$ через выборочные характеристики, т.е. в виде функции от $\mathbf{C}$ и $\widehat{\mathbf{g}}$. Главные части $\mathbf{E} \widehat{\mathbf{k}}^{T} \mathbf{g}$ и $\mathbf{E} \widehat{\mathbf{k}}^{T} \Sigma \widehat{\mathbf{k}}$ представляют интегралы от выборочных функций (по мере $\eta(t)$ ), выражения для которых подготовлены в лемме 4.6 и теореме 4.1. Рассмотрим статистики

$$
\begin{gathered}
\widehat{s}(t)=1-\lambda+N^{-1} \operatorname{tr}(\mathbf{I}+t \mathbf{C})^{-1}, \quad \widehat{\kappa}(t)=t \widehat{\mathbf{g}}^{T} \mathbf{H}(t) \widehat{\mathbf{g}}, \\
\widehat{K}(t, u) \stackrel{\text { def }}{=} t u \widehat{\mathbf{g}}^{T} \mathbf{H}(t) \mathbf{C H}(u) \widehat{\mathbf{g}}=\frac{t \widehat{\kappa}(u)-u \widehat{\kappa}(t)}{t-u}, \quad t, u \geqslant 0, \\
\widehat{\Delta}(t, u)=\widehat{K}(t, u)-(1-\widehat{s}(t)) \widehat{\kappa}(u)-(1-\widehat{s}(u)) \widehat{\kappa}(t)+\widehat{\sigma}^{2}(1-\widehat{s}(t))(1-\widehat{s}(u)),
\end{gathered}
$$

где функция $\widehat{K}(t, u)$ доопределена по непрерьвности при $t=u$. 
Лемма 4.8. При $t \geqslant u \geqslant 0$

$$
s(t) s(u) \mathbf{E} D(t, u)=\mathbf{E} \widehat{\Delta}(t, u)+o, \quad \text { əдe } \quad \mathbf{E}|o| \leqslant \sqrt{M} c_{63} \varepsilon .
$$

Удобно заменить зависимость функционалов от $\eta(t)$ зависимостью от функции $\rho(t)$ вида $\rho(t) \stackrel{\text { def }}{=} \int_{0 \leqslant x \leqslant t} \frac{1}{s(x)} d \eta(x)$. Заметим, что изменение функции $t^{k} \rho(t)$ на $[0, \infty)$ не превьшает $\sqrt{M} \eta_{k+1} \lambda$.

Рассмотрим квадратичный риск (4.3) как функцию $\rho(t): R=\mathbf{E} \Delta^{2}=R(\eta)=R(\rho)$.

TeOpema 4.2. Cтатистика

$$
\widehat{R}=\widehat{R}(\rho)=\widehat{\sigma}^{2}-2 \int\left[\widehat{\kappa}(t)-\widehat{\sigma}^{2}(1-\widehat{s}(t))\right] d \rho(t)+\iint \widehat{\Delta}(t, u) d \rho(t) d \rho(u)
$$

является оценкой $R=R(\rho)$, для которой $\mathbf{E} \widehat{R}(\rho)=R(\rho)+o$, где $|o| \leqslant \sqrt{M} \eta_{8} c_{05} \varepsilon$.

Эта теорема дает оценку, аппроксимируюшую квадратичньй риск с указанной точностью.

Найдем теперь неслучайную главную часть квадратичного риска. Обозначим $K(t, u)=\frac{t \kappa(u)-u \kappa(t)}{t-u}$ и

$$
\Delta(t, u)=K(t, u)-(1-s(t)) \kappa(u)-(1-s(u)) \kappa(t)+\sigma^{2}(1-s(t))(1-s(u)),
$$

где функция $K(t, u)$ доопределена по непрерьвности при $t=u$.

Tеорема 4.3. Квадратичный риск $R=R(\rho)=R_{o}(\rho)+o$, әде

$$
R_{o}(\rho) \stackrel{\text { def }}{=} \sigma^{2}-2 \int s(t) \phi(t s(t)) d \rho(t)+\iint \Delta(t, u) d \rho(t) d \rho(u),
$$

¿де $|o| \leqslant \sqrt{M} \eta_{6} c_{05} \sqrt{\varepsilon}$.

Пусть в частном случае $\eta(x)=\alpha \operatorname{ind}(x \geqslant t)$ при $\alpha, t \geqslant 0, R_{o}(\rho)=R_{o}(t, \alpha)$ ("компрессионно-гребневая регрессия"). Перейдем к пределу при $N \rightarrow \infty$ и $n \rightarrow \infty$, предполагая, что матрищы $\Sigma$ при всех $n$ невырождены и

$$
\lambda=n / N \rightarrow \lambda_{*}<1, \quad \gamma \rightarrow 0, \quad \varepsilon \rightarrow 0, \quad \sigma^{2} \rightarrow \sigma_{*}^{2}, \quad r^{2}=g^{T} \Sigma^{-1} g / \sigma^{2} \rightarrow r_{*}^{2} .
$$

ПримеР 1 . Пусть в этих условиях $\alpha=1$, а $t \rightarrow \infty$ (переход к стандартной нерегуляризованной регрессии в а.р.р.). Тогда

$$
\begin{gathered}
s(t) \rightarrow 1-\lambda_{*}, \quad s^{\prime}(t) \rightarrow 0, \quad \phi(t s(t)) \rightarrow \sigma_{*}^{2} r_{*}^{2}, \\
\kappa(t) \rightarrow \kappa(\infty) \stackrel{\text { def }}{=} \sigma_{*}^{2} r_{*}^{2}\left(1-\lambda_{*}\right)+\sigma_{*}^{2} \lambda_{*}, \quad t \kappa^{\prime}(t) \rightarrow 0 .
\end{gathered}
$$

Квадратичный риск (4.3) стремится к пределу $R^{*}$ такому, что

$$
\lim _{t \rightarrow \infty} \varlimsup_{\substack{N \rightarrow \infty \\ \varepsilon \rightarrow 0}}\left|\mathbf{E} R_{0}(1, t)-R_{*}\right|=0
$$

и

$$
R_{*} \stackrel{\text { def }}{=} \sigma_{*}^{2}\left(1-\lambda_{*}\right)^{-1}\left(1-r_{*}^{2}\right) .
$$

Эта предельная формула получена для нормальных распределений И. С. Енюковым (см. в [31]). Она явно выражает зависимость качества стандартной регрессионной процедуры от размерности наблюдений и объема выборки. 
ПримеР 2. Пусть в тех же условиях при конечном $t$ параметр $\alpha$ выбирается оптимальным образом: $\alpha=\alpha^{0}(t)=s^{2}(t) \phi(t s(t)) / \Delta(t, t)$. Затем $t \rightarrow \infty$. Тогда

$$
R\left(\alpha^{0}, t\right) \rightarrow R_{\infty} \stackrel{\text { def }}{=} \sigma_{*}^{2}\left(1-r_{*}^{2}\right)\left[\lambda_{*}+\left(1-\lambda_{*}\right) r_{*}^{2}\right] /\left[\lambda_{*}\left(1-r_{*}^{2}\right)+\left(1-\lambda_{*}\right) r_{*}^{2}\right]
$$

Величина $R_{\infty} \leqslant \sigma_{*}^{2}\left(1-r_{*}^{2}\right) /\left(1-\lambda_{*}\right)$. При $\lambda_{*} \rightarrow 1$ весовой коэффициент $\alpha^{0} \rightarrow 0$ таким образом, что квадратичный риск остается финитным (стремится к $\sigma_{*}^{2}$ ) несмотря на отсутствие регуляризации.

\section{5. Улучшение линейного дискриминантного анализа}

В [8] была развита предельная теория обобщенных процедур линейных дискриминантных процедур (отличающихся использованием суперпозиции "гребневых оценок" обратной ковариационной матрицы) для анализа $n$-мерных наблюдений при $n \rightarrow \infty$ и $n / N \rightarrow \lambda>0$, где $N$ - объем выборки, в случае двух нормальных совокупностей с совпадающими ковариационными матрицами. Там же было найдено экстремальное предельное решение. В этом разделе мы опишем обобшение этой теории, справедливое при фиксированной размерности наблюдений и фиксированных объемах выборок.

Рассмотрим две $n$-мерные совокупности $\mathscr{S}_{\nu}=\mathbf{N}\left(\mathbf{a}_{\nu}, \Sigma\right), \nu=1,2$, с одинаковыми ковариационньми матрищами $\Sigma$. Пусть $\mathscr{X}_{\nu}=\left\{\mathbf{x}_{m}\right\}, \nu=1,2,-$ выборки (имеется в виду сквозная нумерация векторов выборок) объемов $N_{\nu}>n \geqslant 1$ из совокупностей $\mathscr{S}_{\nu}, \nu=1,2, N=N_{1}+N_{2}, \overline{\mathbf{x}}_{\nu}$ - векторы средних вида $(0.1), \nu=1,2$, и $\mathbf{C}$ - объединенная выборочная ковариационная матрица (1.3). Обозначим $\mathbf{H}=\mathbf{H}(t)=(\mathbf{I}+t \mathbf{C})^{-1}$, $t \geqslant 0$.

Будем изучать рассмотренньй в [8] класс $\mathscr{K}$ линейных дискриминантных функций вида

$$
w(\mathbf{x})=\left(\overline{\mathbf{x}}_{1}-\overline{\mathbf{x}}_{2}\right)^{T} \Gamma(\mathbf{C})\left(\mathbf{x}-\left(\overline{\mathbf{x}}_{1}+\overline{\mathbf{x}}_{2}\right) / 2\right),
$$

где $\Gamma(\mathbf{C})=\int t(\mathbf{I}+t \mathbf{C})^{-1} d \eta(t)-$ матрица, диагонализирующаяся вместе с $\mathbf{C}$, с собственными числами, которые суть интегралы $\int t(1+t \lambda)^{-1} d \eta(t)$, где $\lambda$ - собственные числа С. Относительно $\eta(t)$ предположим, что эта функция имеет ограниченное изменение и достаточное число моментов $\eta_{k}=\int t^{k}|d \eta(t)|, k=1,2 \ldots$.

Пусть дискриминантное правило имеет вид $w(\mathbf{x})>\theta$ против $w(\mathbf{x}) \leqslant \theta$, где $\theta$ - порог классификации. Вероятности ошибок (1.1) зависят от выборок и от параметров $\eta(t)$ и $\theta ;$ обозначим

$$
\alpha(\eta) \stackrel{\text { def }}{=} \min _{\theta}\left(\alpha_{1}+\alpha_{2}\right) / 2=\Phi\left(-J^{1 / 2} / 2\right)
$$

где $J=J(\eta)=G^{2} / D, G=G_{1}-G_{2}$, а условные при фиксированных выборках моменты $G_{\nu}, \nu=1,2$, и условная дисперсия $D$ равны

$$
\begin{gathered}
G_{\nu}=\mathbf{E}\left(w(\mathbf{x}) \mid \mathscr{X}_{1}, \mathscr{X}_{2}, \mathbf{x} \in \mathscr{S}_{1}\right)=\left(\overline{\mathbf{x}}_{1}-\overline{\mathbf{x}}_{2}\right)^{T} \Gamma(\mathbf{C})\left(\mathbf{a}_{\nu}-\left(\overline{\mathbf{x}}_{1}+\overline{\mathbf{x}}_{2}\right) / 2\right), \\
D=\operatorname{var}\left(w(\mathbf{x}) \mid \mathscr{X}_{1}, \mathscr{X}_{2}, \mathbf{x} \in \mathscr{S}_{\nu}\right)=\left(\overline{\mathbf{x}}_{1}-\overline{\mathbf{x}}_{2}\right)^{T} \Gamma(\mathbf{C}) \Sigma \Gamma(\mathbf{C})\left(\overline{\mathbf{x}}_{1}-\overline{\mathbf{x}}_{2}\right), \quad \nu=1,2 .
\end{gathered}
$$


Обозначим

$$
\begin{gathered}
\overline{\mathbf{x}}=\overline{\mathbf{x}}_{1}-\overline{\mathbf{x}}_{2}, \quad k(t)=t \overline{\mathbf{x}}^{T} \mathbf{H}(t) \overline{\mathbf{x}}, \\
g_{\nu}(t)=t\left(\overline{\mathbf{x}}_{1}-\overline{\mathbf{x}}_{2}\right)^{T} \mathbf{H}(t)\left(\mathbf{a}_{\nu}-\left(\overline{\mathbf{x}}_{1}+\overline{\mathbf{x}}_{2}\right) / 2\right), \quad \nu=1,2, \\
d(t, u)=t u \overline{\mathbf{x}}^{T} \mathbf{H}(t) \Sigma \mathbf{H}(u) \overline{\mathbf{x}} .
\end{gathered}
$$

По определению

$$
G_{\nu}=\int g_{\nu}(t) d \eta(t), \quad \nu=1,2, \quad D=\iint d(t, u) d \eta(t) d \eta(u) .
$$

Введем единый размерный параметр

$$
L=\max \left(3\left\|\Sigma_{1}\right\|, 3\left\|\Sigma_{2}\right\|, \mathbf{a}^{2}\right), \quad \text { где вектор } \mathbf{a}=\mathbf{a}_{1}-\mathbf{a}_{2} .
$$

Для компактной записи оценок остаточных членов обозначим $c_{j}=a \max \left(1, L^{j} t^{j}\right)$, $j=1,2, \ldots$, где $a-$ абсолютные константы, $n_{0}=\min \left(N_{1}-1, N_{2}-1\right)$ и $\varepsilon=\sqrt{\gamma+1 / N}$.

Обозначим $y=n / N, y_{\nu}=n / N_{\nu}, \quad \nu=1,2, \quad h(t)=\mathbf{E} n^{-1} \operatorname{tr} \mathbf{H}(t), \quad \mathbf{R}(t)=$ $(\mathbf{I}+t s(t) \Sigma)^{-1}$.

Построим статистики, приближаюшие функции $h(t), s(t), g_{1}(t)$ (оценки $g_{1}(t)$ и $g_{2}(t)$ симметричны) и $d(t, u)$ :

$$
\begin{gathered}
\widehat{h}(t)=n^{-1} \operatorname{tr} \mathbf{H}(t), \quad \widehat{s}(t)=1-t \operatorname{tr}(\mathbf{H}(t) \mathbf{C}) / N, \\
\widehat{g}_{1}(t)=k(t) / 2-(1-\widehat{s}(t)) / \widehat{s}(t), \\
\widehat{d}(t, u)=t u \overline{\mathbf{x}}^{T} \mathbf{H}(t) \mathbf{C H}(u) \overline{\mathbf{x}} .
\end{gathered}
$$

TeOpema 5.1. Прu $0 \leqslant y \leqslant 1 u 0 \leqslant u \leqslant t$

$$
\begin{aligned}
& 1^{\circ} \mathbf{E}(\widehat{h}(t)-h(t))^{2} \leqslant \tau^{2} / N, \mathbf{E}(\widehat{s}(t)-s(t))^{2} \leqslant c_{2} / N ; \\
& 2^{\circ} \mathbf{E} k(t)=t \mathbf{a}^{T}(\mathbf{I}+t s(t) \Sigma)^{-1} \mathbf{a}+\left(y_{1}+y_{2}\right)(1-h(t)) / s(t)+o, \text { дде } o^{2} \leqslant c_{7} \varepsilon^{2} ; \\
& 3^{\circ}(1-y)^{2} \mathbf{E}\left(\widehat{g}_{\nu}(t)-g_{\nu}(t)\right)^{2} \leqslant c_{12} \varepsilon^{2}, \quad \nu=1,2 ; \\
& 4^{\circ}(1-y)^{2} \mathbf{E}[\widehat{d}(t, u) /(\widehat{s}(t) \widehat{s}(u))-d(t, u)]^{2} \leqslant c_{12} \varepsilon^{2} .
\end{aligned}
$$

Решение задачи оценивания первых двух моментов дискриминантной функции дается следуюшим утверждением. Обозначим

$$
\begin{aligned}
\widehat{G}_{\nu} & =\widehat{G}_{\nu}(\eta)=\int \widehat{g}_{\nu}(t) d \eta(t), \quad \nu=1,2, \\
\widehat{D} & =\widehat{D}(\eta)=\iint \frac{\widehat{d}(t, u)}{\widehat{s}(t) \widehat{s}(u)} d \eta(t) d \eta(u) .
\end{aligned}
$$

TEOPEMA 5.2. Прu $0 \leqslant y \leqslant 1$

$1^{\circ}(1-y)^{2}\left(\mathbf{E} \widehat{G}_{\nu}-\mathbf{E} G_{\nu}\right)^{2} \leqslant a \eta_{10} \varepsilon^{2}, \quad \nu=1,2 ;$

$2^{\circ} \operatorname{var} G_{\nu} \leqslant a \eta_{4} / n_{0}, \quad(1-y)^{2} \operatorname{var} \widehat{G}_{\nu} \leqslant a \eta_{4} / n_{0}, \quad \nu=1,2$;

$3^{\circ}(1-y)^{4}(\mathbf{E} \hat{D}-\mathbf{E} D)^{2} \leqslant a \eta_{12} \varepsilon^{2} ;$

$4^{\circ} \operatorname{var} D \leqslant a \eta_{6} / n_{0} u \operatorname{var} \widehat{D} \leqslant a \eta_{4} / n_{0}, \quad \nu=1,2$,

где а - абсолютные константы.

Сконструируем статистику

$$
\widehat{J}(\eta)=\frac{\left[\int\left(k(t)-\left(y_{1}+y_{2}\right)(1-\widehat{h}(t)) / \widehat{s}(t)\right) d \eta(t)\right]^{2}}{\iint[u k(t)-t k(u)] /[\widehat{s}(t) \widehat{s}(u)(u-t)] d \eta(t) d \eta(u)} .
$$


Tеорема 5.3. При $0<y<1 \quad(1-y)^{2} \mathbf{E} D \widehat{D}|\widehat{J}(\eta)-J(\eta)| \leqslant \eta_{9} \varepsilon$.

Эта теорема дает возможность оценивать вероятность ошибки $\alpha(\eta)$ по выборкам с малым смешением и малой дисперсией в условиях а.p.p. Переход к пределу дает предельные формулы работы [8]. Функционал $\widehat{J}(\eta)$ есть отношение двух квадратичных по $\eta(t)$ выражений и допускает очевидную минимизацию. В [8] эта минимизация вьполнена при некоторых дополнительных условиях в предельных формулах и найдены предельные условия экстремальности. Сформулируем этот результат в виде двух теорем.

Рассмотрим в постановке А.Н. Колмогорова последовательность (1.4) задач $\mathscr{P}_{n}$ дискриминантного анализа наблюдений из совокупностей $\mathscr{S}_{\nu}=\mathbf{N}\left(a_{\nu}, \Sigma\right), \nu=1,2$, с дискриминантной функцией (5.1).

Tеорема 5.4. Предположим, что в $\left\{\mathscr{P}_{n}\right\}$

(A) для ковариационных матрии $\Sigma$ выполняются условия теоремы 1.2;

(Б) отношения $y=n / N \rightarrow y^{*}>0, y_{\nu}=n / N_{\nu} \rightarrow y_{\nu}^{*}, \nu=1,2$;

(B) функиии $\mathbf{t a}^{T}(I+t \Sigma)^{-1} \mathbf{a} \rightarrow \phi^{*}(t)$ равномерно при $t \geqslant 0$.

Тогда

$1^{\circ}$ при $n \rightarrow \infty$ равномерно по $t \geqslant 0$ имеет место сходимость $h(t) \rightarrow h^{*}(t)$, $s(t) \rightarrow s^{*}(t)$ и по вероятности $k(t) \rightarrow k^{*}(t)$

$2^{\circ}$ при $n \rightarrow \infty$ имеет место сходимость по вероятности $к$ неслучайныцм пределам $G_{\nu} \rightarrow G_{\nu}^{*}, \nu=1,2, u D \rightarrow D^{*}$

$3^{\circ}$ если $D^{*}>0$, то вероятность ошибки (5.2) сходится по вероятности $\kappa$ $\alpha^{*}=\alpha^{*}(\eta)=\Phi\left(-\left(J^{*}\right)^{1 / 2} / 2\right)$, зде $J^{*}=\left(G_{1}^{*}-G_{2}^{*}\right)^{2} / D^{*}$.

Tеорема 5.5. Предположим, ито в $\left\{\mathscr{P}_{n}\right\}$ выполнены условия (A)-(В) теоремы 5.4 и, кроме того, при каждом п в системе координат, в которой матрица $\Sigma$ диагональна, $\max _{i} a_{i}^{2} / \lambda_{i}<c$, где $a_{i}-$ компонента вектора $\mathbf{a}=\mathbf{a}_{1}-\mathbf{a}_{2}$, соответствующая собственному числу $\lambda_{i}$ матрищь $\Sigma, i=1, \ldots, n$, а с от $n$ не зависит.

Тогда аналитические продолжения функиий $s^{*}(z), \phi^{*}(z)$ u $k^{*}(z)$ удовлетворяют условию Гёльдера на плоскости комплексных z.

Если, кроме того, интегральное уравнение

$$
\int(z+t)^{-1} d \eta(t)=\operatorname{Im}\left(s^{*}(-z) \phi^{*}(-z)\right) / \operatorname{Im} k^{*}(-z)
$$

разрешимо при всех $z>0$, где $\operatorname{Im} k^{*}(-z)>0$, и его решение $\eta(t)=\eta^{o}(t)$ есть функиия ограниченного изменения на $[0, \infty)$, mo $\alpha^{*}\left(\eta^{o}\right)=\inf _{\eta} \alpha^{*}(\eta)$.

ПримеР 1. Предположим, что функция $\eta(t)$ имеет вид единичной ступеньки в точке $t$, a $t \rightarrow \infty$, что соответствует убываюшей "гребневой" регуляризации и переходу к стандартной процедуре. Тогда

$$
\begin{gathered}
h^{*}(t) \rightarrow 0, \quad s^{*}(t) \rightarrow 1, \quad G_{1}^{*} \rightarrow\left(J^{*} / 2-y_{1}^{*}\right) /\left(1-y^{*}\right), \\
D^{*} \rightarrow\left(J^{*}+y_{1}^{*}+y_{2}^{*}\right) /\left(1-y^{*}\right)^{3}
\end{gathered}
$$

и

$$
J^{*} \rightarrow J_{o}^{* 2}\left(1-y^{*}\right) /\left(J_{o}^{*}+y_{1}^{*}+y_{2}^{*}\right),
$$

где $J_{o}^{*}=\lim _{n \rightarrow \infty} \mathbf{a}^{T} \Sigma^{-1} \mathbf{a}$, в согласии с формулой Деева (теорема 1.1). 
ПримеР 2. Пусть предельное распределение собственных чисел матриц $\Sigma$ описывается " $\rho$-моделью" (см. [8]) в зависимости от двух параметров $0 \leqslant \rho<1$ и $\sigma \geqslant 0$. В этом случае предельное спектральное уравнение (1.6) имеет явное аналитическое решение. Пусть $\eta(x)=\operatorname{ind}(x \leqslant t), t \geqslant 0$ (дискриминантная функция с "гребневой регуляризацией"). Пусть при каждом $n$ для всех $i$ отношения $a_{i}^{2} / \lambda_{i}$ равны между собой и равны $J_{n} / n$, где $a_{i}$ - компоненты вектора а в системе координат, где $\Sigma$ диагональна, соответствующие собственным числам $\lambda_{i}$ матрицы $\Sigma$, а $J_{n}=\mathbf{a}^{T} \Sigma^{-1} \mathbf{a}, i=1, \ldots, n$ ("модель одинаковых вкладов"). Тогда при $n \rightarrow \infty$ сушествует предел $J_{o}^{*}=\lim J_{n}$, а предельное значение $J=J(t)$ приобретает вид

$$
J^{*}(t)=J_{o}^{* 2}\left(1-y^{*}+2 y^{*} h^{*}(t)-\left(\rho+y^{*}\right) h^{* 2}(t)\right) /\left(J_{o}^{*}+y_{1}^{*}+y_{2}^{*}\right)
$$

Максимум достигается при $t=t^{o}=\rho y^{*} /\left(\sigma^{2}\left(1-\rho^{2}\right)\right)$ и составляет

$$
J^{*}\left(t^{o}\right)=\max _{t} J^{*}(t)=J_{o}^{* 2}\left(1-\rho y^{*} /\left(\rho+y^{*}\right)\right) /\left(J_{o}^{*}+y_{1}^{*}+y_{2}^{*}\right) .
$$

\section{6. Нормализация при оценке качества многомерных статистических процедур}

В разделах 3-5 мы видели, что ряд функционалов, измеряюших качество многомерных статистических процедур, могут допускать в а.p.p. надежное оценивание исходя из гипотезы о нормальности наблюдений. В этом разделе, следуя [29], мы установим это свойство для пяти классов инвариантных относительно вращений функционалов типа функций качества основных (регуляризованных) многомерных статистических процедур, включая наиболее употребительные.

Относительно совокупностей $\mathscr{S}$ предположим, как и вьшше, что для векторов $\mathbf{x}$ из $\mathscr{S}$ математическое ожидание $\mathbf{E x}=0$ и существуют четвертые моменты всех компонент.

Введем меру "нормализуемости функционалов". Будем говорить, что функция $f: \mathbb{R}^{n} \rightarrow \mathbb{R}^{1}$ случайного аргумента $\mathbf{x} \varepsilon$-нормализуема (везде ниже в среднем квадратичном) для класса совокупностей $\mathscr{K}$, если для каждой совокупности $\mathscr{S} \in \mathscr{K}$ можно указать нормальную случайную величину $\mathbf{y} \sim \mathbf{N}(a, \Sigma)$ с теми же, что у величины $\mathbf{x}$, моментами $\mathbf{a}=\mathbf{E x}$ и $\Sigma=\operatorname{cov}(\mathbf{x}, \mathbf{x})$ и такую, что $\mathbf{E}|f(\mathbf{x})-f(\mathbf{y})|^{2} \leqslant \varepsilon$.

ПримеР 1. Пусть $n=1, \xi \sim \mathbf{N}(0,1)$, функция $f(\mathbf{x})=\mathbf{x}$. Для совокупности, заданной случайной величиной $\mathbf{x}=\xi^{3} / 15$, эта функция $\varepsilon$-нормализуема (величиной $\mathbf{y}=\xi) \mathrm{c} \varepsilon=0.18$.

ПримеР 2. Пусть функция $f(t)$ при $t \geqslant 0$ имеет первую и вторую производную, которые ограничены по абсолютной величине $b_{1}$ и $b_{2}$. Тогда для совокупностей, в которых все переменные имеют первые и четвертые моменты и определен параметр (3.1), функция $f\left(\overline{\mathbf{x}}^{2}\right)$ с $\overline{\mathbf{x}}$ вида (0.1) $\varepsilon$-нормализуема с $\varepsilon=4 b_{1}^{2} d+16 b_{2}^{2} d^{2}$, где $d=\operatorname{var}\left(\overline{\mathbf{x}}^{2}\right) \leqslant$ $M y(2+y) / N, y=n / N$.

ПримеР 3. Для совокупностей, в которых все переменные имеют первые и четвертые моменты и параметры (3.1) и (3.2), согласно теореме 3.4 матричные элементы резольвенты $\mathbf{H}=(\mathbf{I}+t \mathbf{C})^{-1}$ при $t \geqslant 0 \quad \varepsilon$-нормализуемы с $\varepsilon=c_{63}(\gamma+1 / N)$, где $c_{63}$ определено (3.8). 
Исследуем нормализуемость функционалов типа функций качества, построенных с помощью резольвенты выборочных ковариационных матриц вида $\mathbf{S}$ и вида $\mathbf{C}$. Пусть $\mathscr{S}_{1}, \ldots, \mathscr{S}_{k}-n$-мерные совокупности с ожидаемьми значениями $\mathbf{E}_{i} \mathbf{x}=\mathbf{a}_{i}$ и $\operatorname{cov}_{i}(\mathbf{x}, \mathbf{x})=\Sigma_{i}$ и моментами $M_{i}$ вида $(3.1)$ при $\mathbf{x}$ из $\mathscr{S}_{i}, i=1, \ldots, k$. Обозначим $\mathscr{X}_{i}=\left\{\mathbf{x}_{m}\right\}$ выборку из $\mathscr{S}_{i}$ объема $N_{i}, y_{i}=n / N_{i}, \overline{\mathbf{x}}_{i}-$ среднее выборочное,

$$
\begin{aligned}
& \mathbf{S}_{i}=N^{-1} \sum_{m=1}^{N_{i}}\left(\mathbf{x}_{m}-\mathbf{a}_{i}\right)\left(\mathbf{x}_{m}-\mathbf{a}_{i}\right)^{T} \\
& \mathbf{C}_{i}=N^{-1} \sum_{m=1}^{N_{i}}\left(\mathbf{x}_{m}-\overline{\mathbf{x}}_{i}\right)\left(\mathbf{x}_{m}-\overline{\mathbf{x}}_{i}\right)^{T}, \quad i=1, \ldots, k .
\end{aligned}
$$

Рассмотрим следуюшие классы функционалов.

Класс $\mathscr{L}_{1}=\left\{\Phi_{1}(t, \mathbf{A})\right\}$ функционалов вида $n^{-1} \operatorname{tr} \Gamma$ и вида $\overline{\mathbf{x}}_{1}^{T} \Gamma \overline{\mathbf{x}}_{1}$, где $\Gamma=$ $\left(\mathbf{I}+\mathbf{A}+t \mathbf{S}_{1}\right)^{-1}$ или $\Gamma=\left(\mathbf{I}+\mathbf{A}+t \mathbf{C}_{1}\right)^{-1}, 0 \leqslant t<c_{1}$, а $\mathbf{A}-$ неслучайные симметричные неотрицательно определенные матрицы.

Класс $\mathscr{L}_{2}=\left\{\Phi_{2}\left(t_{0}, t_{1}, \ldots, t_{k}\right)\right\}$ функционалов вида

$$
n^{-1} \operatorname{tr} \Gamma \text { и } \overline{\mathbf{x}}_{i}^{T} \Gamma \overline{\mathbf{x}}_{i}, \quad i=1, \ldots, k, \text { при } 0 \leqslant t_{i}<c_{2}, \quad i=0,1, \ldots, k,
$$

где $\Gamma=\left(\mathbf{I}+t_{0} \mathbf{A}+t_{1} \mathbf{S}_{1}+\cdots+t_{k} \mathbf{S}_{k}\right)^{-1}$, либо $\Gamma=\left(\mathbf{I}+t_{0} \mathbf{A}+t_{1} \mathbf{C}_{1}+\cdots+t_{k} \mathbf{C}_{k}\right)^{-1}, \mathrm{a}$ $\mathbf{A}$ - неслучайные симметричные неотрицательно определенные матрищы $n \times n$.

Класс $\mathscr{L}_{3}=\left\{\Phi_{3}\left(t_{0}, t_{1}, \ldots, t_{k}\right)\right\}$ функционалов, представляющих всевозможные (и смешанные) частные производные $\Phi_{2}\left(t_{0}, t_{1}, \ldots, t_{k}\right)$ по аргументам $0<t_{0}, t_{1}, \ldots$, $t_{k}<c_{3}$.

Класс $\mathscr{L}_{4}=\left\{\Phi_{4}\left(\rho_{0}, \rho_{1}, \ldots, \rho_{k}\right)\right\}$ функционалов $n^{-1} \operatorname{tr} \Gamma$, где

$$
\Gamma=\int \Phi_{3}\left(t_{0}, t_{1}, \ldots, t_{k}\right) d \rho_{0}\left(t_{0}\right) d \rho_{1}\left(t_{1}\right) \cdots d \rho_{k}\left(t_{k}\right)
$$

а функции $\rho_{i}(t), i=1, \ldots, k$, определены и имеют ограниченное изменение на $\left[0, c_{4}\right]$.

Класс $\mathscr{L}_{5}=\left\{\Phi_{5}\left(z_{1}, \ldots, z_{k}\right)\right\}$, где $\Phi_{5}-$ дифференцируемые функции $z_{1}, \ldots, z_{m} \mathrm{c}$ производными, ограниченными константой $c_{5}$ по абсолютной величине, и со значениями аргументов, равными функционалам из $\mathscr{L}_{4}$.

Отметим, что к классу $\mathscr{L}_{3}$ принадлежат известные в многомерном статистическом анализе инвариантные относительно вращений функционалы $(\mathbf{a}-\alpha \overline{\mathbf{x}})^{2}$ (где $\mathbf{a}=\mathbf{E x}$, $\alpha$-неслучайньй скаляр $),(\overline{\mathbf{x}}-\mathbf{a})^{T} \Sigma^{-1}(\overline{\mathbf{x}}-\mathbf{a}),(\overline{\mathbf{x}}-\mathbf{a})^{T} \mathbf{S}_{\alpha}^{-1}(\overline{\mathbf{x}}-\mathbf{a})$ и $(\overline{\mathbf{x}}-\mathbf{a})^{T} \mathbf{C}_{\alpha}^{-1}(\overline{\mathbf{x}}-\mathbf{a})$, где (и ниже) $\mathbf{S}_{\alpha}=\mathbf{S}+\alpha \mathbf{I}, \mathbf{C}_{\alpha}=\mathbf{C}+\alpha \mathbf{I}, \alpha>0$ - параметры регуляризации, а также функционалы

$$
\begin{aligned}
& n^{-1} \operatorname{tr}\left(\mathbf{S}_{\alpha}^{-1}-\Sigma^{-1}\right)^{2}, \quad n^{-1} \operatorname{tr}\left(\mathbf{C}_{\alpha}^{-1}-\Sigma^{-1}\right)^{2}, \quad n^{-1} \operatorname{tr}\left(\mathbf{I}-\mathbf{S}_{\alpha}^{-1} \Sigma\right)^{2}, \\
& n^{-1} \operatorname{tr}\left(\mathbf{I}-\mathbf{C}_{\alpha}^{-1} \Sigma\right)^{2}, \quad(\overline{\mathbf{x}}-\mathbf{a})^{T} \mathbf{C}_{\alpha}^{-1} \Sigma \mathbf{C}_{\alpha}^{-1}(\overline{\mathbf{x}}-\mathbf{a}), \quad\left(\overline{\mathbf{x}}_{1}-\overline{\mathbf{x}}_{2}\right)^{T} \mathbf{C}_{12}^{-1}\left(\overline{\mathbf{x}}_{1}-\overline{\mathbf{x}}_{2}\right),
\end{aligned}
$$

где $\mathbf{C}_{12}=\alpha_{0} \mathbf{I}+\alpha_{1} \mathbf{C}_{1}+\alpha_{2} \mathbf{C}_{2}, \alpha_{0}, \alpha_{1}, \alpha_{2}>0$ (последние функционалы возникают в "гребневом" линейном дискриминантном анализе). К классу $\mathscr{L}_{4}$ принадлежат функционалы, измеряющие качество ряда процедур с “обобщенной гребневой оценкой” обратной ковариационной матрищы (см. [7; гл. 2]). 
Teорема 6.1. Пусть $\mathscr{P}=\left\{\mathscr{P}_{n}\right\}-$ последовательность задач

$$
\mathscr{P}_{n}=\left(\mathscr{S}_{\nu}, \mathbf{a}_{\nu}, M_{\nu}, N_{\nu}, \nu=1, \ldots, k ; \Phi_{i}, i=1, \ldots, 5\right)_{n}, \quad n=1,2, \ldots
$$

анализа наблюдений $\mathbf{x} \in \mathbb{R}^{n}$ из совокупностей $\mathscr{S}_{\nu} c \mathbf{E x}=\mathbf{a}_{\nu}$ и моментами $M_{\nu}$ вида (3.1), $\nu=1, \ldots, k$. Пусть константы $c_{1}-c_{5}$ и $k$ от $n$ не зависят. Предположим, что при всех $\nu=1, \ldots, k$ и $n \rightarrow \infty$

(A) величины $M_{\nu}$ и $\mathbf{a}_{\nu}^{2}$ равномерно ограничены;

(Б) величины (3.2) для совокупностей $\mathscr{S}_{\nu}$ стремятся к нулю;

(В) отношения $n / N_{\nu}$ равномерно ограничень;

(Г) изменение функиий $\rho_{\nu}(t)$ на $\left[0, c_{4}\right]$ равномерно ограничено.

Тогда функииональ из классов $\mathscr{L}_{1}-\mathscr{L}_{5}$ в-нормализуемь $с \varepsilon \rightarrow 0$.

\section{Обсуждение}

Таким образом, за последние годы в математической статистике развит новый метод асимптотического исследования (асимптотика растущей размерности наблюдений, а.р.p.), которьй учитывает специфику явлений при оценивании большого числа параметров, сопоставимого с объемами выборок, и в котором существенную роль играет отношение размерности наблюдений к объему выборки. Его можно назвать теорией существенно многомерных, или, точнее, существенно многопараметрических, эффектов. Эти эффекты возникают вследствие дополнительного усреднения ("самоусреднения", "перемешивания"), которое дает большое число слабо зависимых переменных. Дополнительное усреднение делает функции многих переменных малочувствительными к деталям распределений, и главные члены а.p.p. оказываются зависимьми только от первых двух моментов переменных. В этом смысле сушественно многомерные задачи оказываются "нормализуемыми”. Описанный подход можно назвать теорией статистического анализа по первым двум (истинным и выборочным) моментам переменных в условиях ограниченной зависимости большого числа переменных.

Условия ограниченной зависимости, достаточное для применения развитого подхода, определяются двумя параметрами: максимальным четвертым моментом $M$ проекции векторов наблюдений на неслучайные оси (3.1) и мерой $\gamma$ дисперсии квадратичных форм вида (3.2). Для нормальных распределений $\left(0_{n}, \Sigma_{n}\right)$ момент $M=3\left\|\Sigma_{n}\right\|^{2}$, а $\gamma=2 \operatorname{tr} \Sigma_{n}^{2} / 3\left\|\Sigma_{n}\right\|^{2} \quad\left(\left\|\Sigma_{n}\right\|-\right.$ спектральная норма). Для независимых компонент $\mathbf{x}$ величина $\gamma=O\left(n^{-1}\right)$.

В отличие от асимптотического подхода [3]-[6], [8], [10]-[15] и [18]-[26] в разделах 3-6 развита теория выделения главных частей асимптотики, применимая при любой фиксированной размерности наблюдений $n$ и любых фиксированных объемах выборок $N$. Остаточные члены асимптотики оцениваются сверху с точностью до абсолютных констант. Малость этих членов гарантируется при ограниченных моментах $M$, ограниченных отношениях $n / N$, больших $N$ и малых $\gamma$. Тем самым, результаты, полученные в серии предыдуших работ по многомерному статистическому анализу в предположении о нормальности, в частности, [3]-[6], [11]-[13], [16], [32] и [33], допускают возможность распространения на распределения из более широкого класса. Погрешность такого расширения области применимости оценивается сверху с помошью методики раздела 6. 
Проблема устойчивости оценок к расширению класса совокупностей (к “загрязнению" выборки) привлекла внимание после известных работ Тьюки 1960 г. по устойчивости оценок параметра масштаба, которые привели к появлению серии исследований по построению разнообразных "робастных" оценок. Отметим, что до сих пор известен только один класс многомерных устойчивых оценок, называемых экспоненциально взвешенными [34]; [7], гл. 8. Эти оценки, однако, с ростом размерности быстро утрачивают эффективность, что связано с отсутствием предположений, ограничивающих свойства загрязнений. Полученные результаты позволяют дополнить известные робастные методы для задач ограниченной размерности сушественно многомерными методами. В тех случаях, когда класс загрязнений априори ограничивается распределениями с ограниченными моментами $M$ и малыми $\gamma$, найденные вьше сушественно многомерные решения оказываются заведомо робастными. Устойчивость в них достигается благодаря регуляризации процедур и нечувствительностью к высшим моментам.

Асимптотические методы статистики, ориентированные ранее, в основном, на одномерные задачи, получают дополнительньй математический аппарат для учета существенно многомерных явлений. Его характерная особенность состоит во введении множественного описания переменных в форме эмпирических функций распределения, которые представляют большое число ограниченно зависимых переменных. Функционалы, построенные по эмпирическим распределениям, приближенно нормализуются и в этом смысле становятся независимыми от распределений. В число таких функционалов входят стандартные функции качества регуляризованных модификаций наиболее распространенных процедур. В результате открывается возможность построения $\varepsilon$-неулучшаемых многомерных статистических процедур, свободных от гипотез о распределениях.

Развит следующий общий подход к решению многомерных статистических задач.

1. Выводятся уравнения, связывающие главные в а.р.p. части функционалов, зависящих от параметров, и функционалов, зависящих от оценок (в частности, спектральные уравнения), и оценивается сверху дисперсия функционалов. Эти уравнения используются для определения спектральных свойств неизвестных ковариационных матрищ (раздел 3), для уточнения оценок, для стабилизации и уточнения многомерных статистических процедур (разделы 4 и 5). Малость дисперсии функционалов, измеряющих качество статистических процедур в а.p.p., дает возможность надежно оценивать качество процедур и гарантировать их улучшение.

2. Вводится класс обобшенных многомерных статистических процедур (алгоритмов), зависимых от априорных параметров или функций.

3. Выделяются в а.p.p. главные части функции качества этих обобщенных процедур и выражаются, с одной стороны, через параметры совокупностей (что имеет теоретическое значение) и, с другой стороны, в виде функций только от статистик (для приложений).

4. Решается экстремальная задача для главной части функции качества и вьводится условие экстремальности процедуры в классе.

5. Оценивается погрешность, с которой построенная процедура является неулучшаемой.

Возникает, однако, вопрос о точности асимптотических выражений и оценок качества. Остаточные члены a.p.p. имеют порядок малости $\sqrt{\gamma+1 / N}$, где в лучшем случае $\gamma=O\left(n^{-1}\right)$. При попытке улучшения решений возникает необходимость восстановления функций распределения параметров по распределению оценок, и точ- 
ность уменьшается. Здесь во всех случаях необходима дополнительная регуляризация: при решении плохо обусловленных обратных задач, при использовании производных от эмпирических функций, при решении интегральных уравнений Фредгольма первого рода. Природа этих трудностей здесь та же, что и в классической задаче оценивания функции распределения. Требуется усреднение эмпирических функций с достаточно малым интервалом, чтобы выделить закономерность, но с достаточно большим, чтобы эта закономерность была выделена надежно. В результате погрешность модифицированных процедур может возрасти до $O\left(n^{-\alpha}\right)$, где $\alpha>0$ мало (в [35] $\alpha=1 / 4)$.

Можно сделать общий вьвод: рассмотренньй подход позволяет предложить независимые от распределений надежные оценки качества ряда многомерных статистических процедур и путь, на котором можно искать улучшенные и, возможно, приближенно неулучшаемые решения. На этом направлении сделаны только первые шаги.

В заключение хочу искренне поблагодарить Л. Д. Мешалкина за внимание к работе и предложения, которые способствовали улучшению рукописи по существу. Хочу также поблагодарить В. М. Бухштабера за обсуждение результатов решения дискриминантной задачи и неизменную поддержку.

\section{СПИСОК ЛИТЕРАТУРЫ}

[1] Ибрагимов И. А., Хасьминский Р.З. Асимптотическая теория оценивания. М.: Наука, 1979.

[2] Закс Ш. Теория статистических выводов. М.: Мир, 1975.

[3] Деев А. Д. Представление статистик дискриминантного анализа и асимптотическое разложение при размерности пространства, сравнимой по величине с объемом выборки // Докл. АН СССР. 1970. Т. 195. С. 759-762.

[4] Деев А. Д. О дискриминантной функции, построенной по независимым блокам переменных // Изв. АН СССР. Сер. Техн. киберн. 1974. № 4. С. 204-207.

[5] Архаров Л.В. Предельные теоремы для характеристических корней выборочной ковариационной матрицы // Докл. АН СССР. 1971. Т. 199. С. 994-997.

[6] Мешалкин Л.Д. Приписывание числовых значений качественным признакам // Статистические системы управления (Вильнюс). 1976. Т. 14. С. 49-55.

[7] Айвазян С. А., Бухштабер В. М., Енюков И. С., Мешалкин Л. Д. Прикладная статистика: классификация и снижение размерности. М.: Финансы и статистика, 1989.

[8] Сердобольский В. И. О минимальной вероятности ошибки в дискриминантном анализе // Докл. АН СССР. 1983. Т. 270. С. 1066-1070.

[9] Wald A. On a statistical problem arising in the classification of an individual into one of two groups // Math. Statist. 1940. V. 15. P. 147-163.

[10] Мешалкин Л. Д., Сердобольский В. И. Ошибки при классификации многомерных наблюдений // Теория вероятн. и ее примен. 1978. Т. 23. С. $772-781$.

[11] Заруцкий В. И.О классификации нормальных векторов простой структуры зависимостей в пространстве большой размерности // Теория вероятн. и ее примен. 1978. Т. 23. C. $473-475$.

[12] Сердобольский В.И. Об ошибках классификикации по выборочным данным // Теория вероятн. и ее примен. 1979. Т. 24. С. 130-143.

[13] Yin Q., Krishnaiah P. R. (1) On limit of the largest eigenvalue of the large-dimensional sample covariance matrix // Prob. Theory Related Fields. 1988. V. 78. P. 509-521.

[14] Сердобольский В. И. Дискриминантный анализ наблюдений большой размерности. М.: Научный совет по комплексной проблеме "кибернетика" АН СССР, 1979.

[15] Сердобольский В.И. Дискриминантный анализ при большом числе переменных // Докл. АН СССР. 1980. Т. 254. С. 39-44.

[16] Андерсон Т. Введение в многомерный статистический анализ. М.: Физматгиз, 1963.

[17] Уилкс С. Математическая статистика. М.: Наука, 1967. 
[18] Марченко В.А., Пастур Л.А. Распределение собственных значений в некоторых ансамблях случайных матриц // Матем. сб. 1967. Т. 72. С. 507-536.

[19] Гирко В. Л. Теория случайных матриц. Киев: Вища школа, 1975.

[20] Гирко В. Л. Спектральная теория случайных матриш. М.: Наука, 1988.

[21] Bai Z. D. Convergence rate of the expected spectral distribution of large random matrices // Ann. Probab. 1993. V. 21. P. 649-672.

[22] Silverstein J. W., Bai Z.D. On the emirical distribution of eigenvalues of a class of large-dimensional random matrices // J. Multivariate Anal. 1995. V. 54. P. 175-192.

[23] Сердобольский В. И. Резольвента и спектральные функции выборочных ковариационных матриц растущей размерности // УМН. 1985. Т. 40. С. 197-198.

[24] Гирко В. Л. Введение в общий статистический анализ // Теория вероятн. и ее примен. 1987. T. 32. C. $252-265$.

[25] Girko V. L. General equation for the eigenvalues of empirical covariance matrices I, II // Random Oper. and Stoch. Equ. 1994. V. 2. № 1. P. 13-25; № 2. P. 175-187.

[26] Гирко В. Л. Многомерньй статистический анализ. Киев: Вища школа, 1988; Girko V. L. Statistical Analysis of Observations of Increasing Dimension. Dordrecht: Kluwer Acad. Publ., 1995.

[27] Сердобольский В.И. О точности асимптотических выражений для спектральных функций эмпирических ковариационных матриц // УМН. 1994. Т. 49. С. 173-174.

[28] Сердобольский В.И.Спектральные свойства выборочных ковариационных матриц // Теория вероятн. и ее примен. 1995. Т. 40. С. 910-918.

[29] Сердобольский В.И. Нормализация функционалов качества многомерных статистических процедур // Докл. РАН. 1995. Т. 343. С. 743-745.

[30] Ширяев А. Н. Вероятность. М.: Наука, 1970.

[31] Айвазян С. А., Бухштабер В. М., Енюков И. С., Мешалкин Л. Д. Прикладная статистика: исследование зависимостей. М.: Финансы и статистика, 1985.

[32] Барсов Д. А. Оптимальные гребневые оценки ковариационной матрицы в дискриминантном анализе большой размерности // Теория вероятн. и ее примен. 1982. Т. 27. С. 820-821.

[33] Баранова Т. А. Асимптотическая невязка линейной обобщенной гребневой регрессии // Пятая международн. Вильнюсская конф. по теории вероятн. и матем. статистике. Тезисы докладов. Т. 3. Вильнюс: ИМК АН Лит. ССР, 1989. С. 46-47.

[34] Meshalkin L. D. Some mathematical methods for the study of non-communicable diseases // Proc. 6th Intern. Meeting of Intern. Epidemiological Assoc., Primosten (Yugoslavia). Aug. 29, 1971.

[35] Сердобольский В. И. Асимптотически доминирующее оценивание векторов математических ожиданий // Теория вероятн. и ее примен. 1999. Т. 44. № 1. 